\title{
A SECOND-ORDER MULTI-FLUID MODEL FOR EVAPORATING SPRAYS
}

\author{
Guillaume Dufour $^{1,2}$ And Philippe Villedieu ${ }^{1,2}$
}

\begin{abstract}
The aim of this paper is to present a method using both the ideas of sectional approach and moment methods in order to accurately simulate evaporation phenomena in gas-droplets flows. Using the underlying kinetic interpretation of the sectional method [Y. Tambour, Combust. Flame 60 (1985) 15-28] exposed in [F. Laurent and M. Massot, Combust. Theory Model. 5 (2001) 537-572], we propose an extension of this approach based on a more accurate representation of the droplet size number density in each section ensuring the exact conservation of two moments (as opposed to only one moment used in the classical approach). A corresponding second-order numerical scheme, with respect to space and droplet size variables, is also introduced and can be proved to be positive and to satisfy a maximum principle on the velocity and the mean droplet mass under a suitable CFL-like condition. Numerical simulations have been performed and the results confirm the accuracy of this new method even when a very coarse mesh for the droplet size variable (i.e.: a low number of sections) is used.
\end{abstract}

Mathematics Subject Classification. 35Q35, 65Z05, 76T10.

Received: October 14, 2004. Revised: April 8, 2005.

\section{INTRODUCTION}

Sprays - a dispersed phase of liquid droplets in a continuous gaseous phase - play an important role in a wide range of applications such as combustion, spray drying, evaporative cooling, agricultural spraying and many other domains. In these gas-droplet flows, the polydisperse aspect of the spray has a strong effect since the droplet dynamics depends on their inertia, thus on their size. For example, in the combustion domain, the size distribution of the droplets can have a significant influence on the flame structure. Among all the phenomena acting on the size distribution of the spray, secondary breakup, coalescence and evaporation are the most known examples.

At the kinetic level, the liquid phase is described by a probability density function (p.d.f.) $f$, the quantity $f(t, x, s, v) \mathrm{d} x \mathrm{~d} s \mathrm{~d} v$ denoting the average number of droplets at time $t$ located in a volume $\mathrm{d} x$ around $x$ with a size in a $d s$-neighbourhood of $s$ and a velocity in a $\mathrm{d} v$-neighbourhood of $v$. Focusing on the evaporation phenomenon and neglecting the effects of coalescence and secondary breakup, the evolution of the spray may be predicted by the following Boltzmann-type equation, where $K$ denotes the evaporation rate and $A$ the force

\footnotetext{
Keywords and phrases. Moment method, sectional method, sprays, evaporation, multi-fluid.

${ }^{1}$ Laboratoire MIP, Université Paul Sabatier, 118 Route de Narbonne, 31062 Toulouse Cedex, France. dufour@mip.ups-tlse.fr

2 DMAE/Onera - 2, Avenue E. Belin, B.P 4025, 31055 Toulouse Cedex, France. Philippe.Villedieu@onera.fr
} 
exerted on the droplets per mass unit (see for example $[39,40]$ ):

$$
\partial_{t} f+v \cdot \nabla_{x} f+\nabla_{v} .(A f)-\partial_{s}(K f)=0 .
$$

Usually, in all methods developed to simulate the evolution of gas-droplets flows, the behaviour of the continuous phase (the gas) is almost uniquely modeled using the Navier-Stokes equations and is numerically solved using a finite volume method. However, to solve the evolution of the dispersed phase (droplets), mainly two approaches have been proposed. On one hand, the Lagrangian method relies on a direct discretization of equation (0.1). Considering the size of the phase space, this can be done only using a stochastic particle method $[9,15,20,30,33]$. Such a method has been proven to be efficient and accurate but also to be expensive in terms of memory requirements and CPU time as a huge number of "numerical parcels" is required for accurate unsteady computation. On the other hand, the Eulerian method is based on the derivation of a set of averaged conservation equations for some moments of the distribution function $f$. This approach is numerically very competitive but suffers from severe physical limitations due to the intrinsic poor description of the droplet size distribution. In particular, breakup and coalescence cannot be taken into account in the standard two-fluid approach where only two moments in size of the distribution function are resolved (droplet number and droplet mass density). Some other Moment Methods have been developed (see for example $[1,2,32,41]$ ) and recently, Beck and Watkins proposed a real improvement on this kind of method, based on conservation equations for several droplet size moments allowing the development of spray submodels and the possibility to take into account some complex phenomena such as heat transfer, breakup and collision [3,4]. However, such a method still needs to presuppose a global shape for the whole size distribution of the spray, introducing some limitations. In particular, when considering the coupling between the size of the droplets and their velocity, the shape of the joint distribution has to be prescribed arbitrarily.

Another attempt at deriving a Eulerian model for polydispersed sprays was developed by Tambour et al. $[18,37]$. The idea was to consider the dispersed phase as a set of continuous media called "fluids", each "fluid" corresponding to a statistical average between two fixed sizes: a section. More recently, Laurent and Massot provided a rigorous kinetic framework for dilute sprays in laminar flames [24] as well as for dense sprays with coalescence [25] and denoted this method as a "multi-fluid" one. This method was shown to apply to realistic cases with a limited number of sections if an appropriate discretization of the size phase space is used, combined with a particular treatment for the last section, involving an exponential approximation of the droplet density function [26]. However, this approach was shown to be in general only first order accurate relative to the droplet size variable [22] thus resulting in strong numerical diffusion when too few sections are used. The problem of finding an appropriate way to improve the accuracy of the method and also minimizing the number of sections thus becomes critical, especially when considering industrial codes which are intended to perform complex three-dimensional simulations. Some kind of answer is currently being studied by Laurent [23] where the choice of the approximation function for the number density distribution in every section is discussed.

In this article, a method combining both ideas of moment and multi-fluid methods is presented. The aim of this work is to provide a multi-fluid-type method which allows the use of only a small number of sections for numerical simulations. To this end, an appropriate set of moments of the number density functions is chosen to be preserved and an accurate approximation of the number density function involving piecewise exponential functions is proposed. The key point is that this approximation is chosen under the constraint of preserving a given set of moments of the number density function, thus improving the accuracy of the classical multi-fluid method up to the second order in all variables. Using numerical simulations, the choice of both the set of moments and the shape of the approximation function is also shown to be well-suited for such polydispersed two-phase flows, thus providing at least the correct evolution trend even when a coarse mesh in the size space is used.

The paper is organized as follows: in a first step, it will be explained how the multi-fluid approach can be re-interpreted using moment methods in order to improve its accuracy. The new model obtained this way may also be seen as an extension of the standard two-fluid model, since using only one section in the new approach yields a two-fluid type model. In a second step, the numerical algorithm used to solve the resulting set of 
equations will be described, especially the evaporation scheme involving the kinetic interpretation introduced in the first part. In the third part, some theoretical results about the consistency of the scheme and the accuracy of the model will be given and finally in the fourth part some numerical results illustrating the accuracy of this new approach will be presented.

\section{An improvement FOR THE MULTi-FLUID MODEL}

\subsection{The classical multi-fluid model}

\subsubsection{Kinetic description of the spray}

Since we are dealing with polydispersed sprays, a parameter to describe the size of the droplets will be needed. All the droplets are assumed to be spherical so that the size of a given droplet is fully characterized by any of the following quantity: its radius, surface or volume. In the following, the quantity $s$ will denote the surface of the droplets, taken as the size variable.

For the sake of simplicity, but without any loss of generality, we consider that the forces exerted on the droplets are only due to their interaction with the fluid (the drag force) and the effect of gravity is neglected. Moreover, the evaporation law considered is the $d^{2}$-law without convective correction term and the drag force is assumed to be given by a Stokes law. Let $A$ be the Stokes drag force per mass unit due to the difference between the droplet velocity and the surrounding gas one $U_{g}$. Its expression reads:

$$
A(t, x, s, v)=\frac{U_{g}(t, x)-v}{\tau(s)}
$$

where $\tau(s)$ is the mean time it takes for a droplet of size $s$ to adapt to the velocity of the gas. The Stokes law assumes that this time is directly proportional to the surface of the droplet. More precisely we have for a droplet of surface $s$ and mass density $\rho_{l}$ embedded in a gas of viscosity $\mu_{g}$ :

$$
\tau(s)=\frac{\rho_{l} s}{18 \pi \mu_{g}}
$$

For more sophisticated models of the forces exerted by a carrier phase on a droplet, the reader may refer to [29] and for a general review to [8].

Concerning the evaporation phenomenon, the $d^{2}$-law states that the surface of a droplet decreases linearly with time. If $s(t)$ denotes the surface of a droplet as a function of time we have:

$$
\frac{\mathrm{d} s}{\mathrm{~d} t}=-K=\mathrm{C}^{\mathrm{te}}<0
$$

with $K$ being the evaporation rate. For the sake of simplicity we did not take into account the temperature variable in this study. However, the method described in this paper may easily be extended in the more general case. Moreover, more complex evaporation laws could be considered ( $K$ depending on the surface, the temperature and the velocity of the droplets) by assuming that the evaporation rate is locally constant $[14,16,30]$.

\subsubsection{Semi-kinetic model}

The semi-kinetic model [24] eliminates the velocity variable by replacing the kinetic equation (0.1) with two equations on the moments $n$ and $n \bar{v}$ which are respectively the moments of $f$ of order 0 and 1 with respect to the velocity variable. Practically, starting from the full kinetic description of the spray, we deduce evolution 
equations for the quantities

$$
\begin{aligned}
n(t, x, s) & =\int_{v} f(t, x, s, v) \mathrm{d} v \\
n(t, x, s) \bar{v}(t, x, s) & =\int_{v} v f(t, x, s, v) \mathrm{d} v
\end{aligned}
$$

by multiplying Equation (0.1) respectively by 1 and $v$ and integrating over the whole velocity phase space. If the number density function is assumed to rapidly decrease to zero for large droplet velocities, we obtain the following system:

where

$$
\left\{\begin{array}{r}
\partial_{t} n+\nabla_{x} \cdot(n \bar{v})-\partial_{s}(K n)=0 \\
\partial_{t}(n \bar{v})+\nabla_{x} \cdot(n \bar{v} \otimes \bar{v})+\nabla_{x} \cdot P-n \bar{A}-\partial_{s}(K n \bar{v})=0
\end{array}\right.
$$

$$
\bar{A}=\frac{U_{g}(t, x)-\bar{v}}{\tau(s)} .
$$

$P$ may be seen as a kinetic stress tensor, related to the velocity dispersion (see also [27]), and is defined by:

$$
P=\int_{v} f(v-\bar{v}) \otimes(v-\bar{v}) \mathrm{d} v .
$$

Remark 1.1. Physically, $n$ can be interpreted as the droplet number density. We will also use the mass density which is defined as:

$$
m(t, x, s)=\int_{v} \frac{\rho_{l} s^{3 / 2}}{3 \sqrt{4 \pi}} f(t, x, s, v) \mathrm{d} v .
$$

This model is obviously not closed since we lack an expression for $P$ depending only on $n$ and $\bar{v}$. One may note that in general, using such a method for obtaining an evolution equation on the $p$-th moment of $f$ requires an information on the $(p+1)$-th moment. In order to close the model $(1.4)$, we either have to give a constitutive law for this pressure equivalent term (see for example [35]) or to assume a particular form for the p.d.f. $f$ $[17,27]$. This closure aspect will be discussed in the next section when we will present the multi-fluid model (see also $[24,25])$.

Remark 1.2. One may repeat the same process on the size variable and obtain a moment model. Using only one moment in size gives a simplified model which yields low computational cost but is not able to take into account the evaporation phenomenon since it provides no information on the mean size of the droplets. Therefore, some methods using several moments in size have been developed, allowing a more accurate description of the spray's physical behaviour (see for example $[1-4,32,41]$ ). The multi-fluid approach is based on a different point of view, which consists in first discretizing the size space before performing any average in size.

\subsubsection{Multi-fluid model}

In this part we follow the main lines of [24]. Let $N$ be a fixed positive integer, we choose a finite increasing series of droplet sizes $\left(s_{i}\right)_{0 \leq i \leq N}$ defining $N$ finite intervals of size $I_{i}=\left[s_{i-1}, s_{i}\right.$ [ and one infinite interval $I_{N+1}=\left[s_{N},+\infty[\right.$. As stated before, the size $s$ of the droplets denotes its surface. Following the original terminology of Tambour $[18,37]$ the term "section" will denote any interval of size. In any section, the number density function is assumed to have the following simple structure:

$$
n(t, x, s)=n_{i}(t, x) \kappa_{i}(s) \quad \text { for } s_{i-1} \leq s<s_{i}
$$

so that the profile of $n$ in a given section is fixed as a function only of the size of the droplet and does not depend on $t$ and $x$. Such an assumption is quite natural since the principle of the sectional approach is to take some moments at a given size of the distribution function with respect to the velocity variable. 
Moreover, in order to close our system, we make an a priori assumption on the droplet velocity distribution function which consists in supposing that all droplets in a same section have the same velocity. This hypothesis is equivalent to assume that there is no velocity dispersion and then that the number density function $f$ reads:

$$
f(t, x, s, v)=\sum_{i=1}^{N+1} \mathbb{I}_{s_{i-1} \leq s<s_{i}}(s) \cdot n_{i}(t, x) \cdot \delta_{v-\bar{v}_{i}(t, x)}(v) \cdot \kappa_{i}(s) .
$$

Assuming the particular form of the density function, the pressure term $\nabla_{x} . P$ vanishes in the system (1.4) and we obtain:

$$
\left\{\begin{array}{r}
\partial_{t} n+\nabla_{x} \cdot(n \bar{v})-\partial_{s}(K n)=0 \\
\partial_{t}(n \bar{v})+\nabla_{x} \cdot(n \bar{v} \otimes \bar{v})-n \bar{A}-\partial_{s}(K n \bar{v})=0
\end{array}\right.
$$

Remark 1.3. We may also have given a particular model for the pressure equivalent term $P$, also performing a closure for the semi-kinetic model but as explained in [24], choosing the velocity dispersion to be zero enables us to have mean values for the evaporation rate or the drag force term depending only on the mean velocity in the section. In particular we may write $\bar{A}=A(\bar{v})$, and this property also holds in the case where $K$ depends on the velocity.

At this point, only the profile of the distribution within a section remains to be prescribed. In the classical multi-fluid model, it is assumed that in any finite section the function $\kappa_{i}$ is constant and this constant is denoted $\alpha_{i}$. As it is noted in [24], such a choice is completely arbitrary and is related to the structure of the sectional approach which presupposes a shape of the distribution function inside a section which is independent of the state of the spray outside the section. We have to emphasize the fact that, as developed in [25] the multi-fluid model takes for size variable the radius of the droplets $r$. Hence, $\kappa_{i}$ is chosen constant as a function of the radius (see Rems. 1.4 and 1.5) and is determined using the conservation of the mass density.

These last two points addressed, the way to derive the multi-fluid equations is the following: in any section, multiply both equations by $\frac{4 \pi \rho_{l}}{3} r^{3}$ where $\rho_{l}$ is the density of the liquid, integrate them over the whole section and use an "upwind" approximation for the evaporation fluxes $(K n$ and $K n \bar{v})$. This yields $2 N+2$ equations which may be written, for $1 \leq i \leq N+1$ :

$$
\left\{\begin{array}{c}
\partial_{t} m_{i}+\partial_{x}\left(m_{i} \bar{v}_{i}\right)+\left(E_{i}^{(1)}+E_{i}^{(2)}\right) m_{i}-E_{i+1}^{(1)} m_{i+1}=0 \\
\partial_{t}\left(m_{i} \bar{v}_{i}\right)+\partial_{x}\left(m_{i} \bar{v}_{i} \otimes \bar{v}_{i}\right)+\left(E_{i}^{(1)}+E_{i}^{(2)}\right) m_{i} \bar{v}_{i}-E_{i+1}^{(1)} m_{i+1} \bar{v}_{i+1}=m_{i} \overline{A_{i}}
\end{array}\right.
$$

where:

$$
\begin{gathered}
E_{i}^{(1)}=-\frac{4 \pi}{3} \rho_{l} r_{i} K \kappa_{i}\left(r_{i}\right), \quad E_{i}^{(2)}=-\int_{r_{i-1}}^{r_{i}} \frac{4 \pi}{3} \rho_{l} K \kappa_{i}(r) \mathrm{d} r \\
\overline{A_{i}}=\int_{r_{i-1}}^{r_{i}} \frac{4 \pi}{3} \rho_{l} r^{3} A\left(t, x, r, \bar{v}_{i}\right) \kappa_{i}(r) \mathrm{d} r
\end{gathered}
$$

with the convention that $E_{N+2}=0, r_{N+1}=+\infty$ and where $m_{i}(t, x)=\int_{r_{i-1}} \frac{r_{i}}{3} \frac{4 \pi}{3} \rho_{l} r^{3} n_{i}(t, x) \alpha_{i} \mathrm{~d} r$ is the mass density in the the section $i$.

Remark 1.4. The profile of the distribution may be written using any variable size. If $\kappa_{i}$, as a function of the radius $r$, is equal to a constant $\alpha_{i}$ in the section $\left[r_{i-1}, r_{i}\right.$ [ we have for $s$ and $V$ denoting respectively the surface and the volume of the droplets, by noting that $\kappa_{i}(r) \mathrm{d} r=\kappa_{i}(s) \mathrm{d} s=\kappa_{i}(v) \mathrm{d} v$ :

$$
\kappa_{i}(r)=\alpha_{i}, \quad \kappa_{i}(s)=\frac{\alpha_{i}}{2 \sqrt{4 \pi s}}, \quad \kappa_{i}(V)=\frac{\alpha_{i}}{(4 \pi)^{1 / 3}(3 V)^{2 / 3}} .
$$

Remark 1.5. It has to be emphasized that, for optimal accuracy, the discretization of the size space has to be chosen carefully depending on the choice of the size variable. This aspect will not be developed here and, 
for a study on this subject in the case of noncolliding evaporating sprays the reader may refer to [12] or, for a complete numerical analysis, to [22].

\subsection{Improving the multi-fluid model}

Studying the whole process of obtaining the multi-fluid model from the kinetic equation on $f$, one may note that after the discretization of the size space, one of the most important parameter of the sectional approach lies within the choice of $\left(\kappa_{i}\right)_{i}$, which can be interpreted as an approximation of the density function in the $i$-th section. With such an idea, an improvement of the method would logically be to choose some affine function for $\kappa$ in order to have a better approximation. But this approach yields two major problems. First, limiters on the slopes are needed in order to ensure that the approximate density remains positive. Secondly, such an approach is ill-adapted if a very small number of sections is used, since it is difficult to approach accurately the asymptotic behaviour of the distribution function, for large values of the droplet size, with piecewise affine functions. We propose here a different approach, choosing a two parameters representant for the distribution function in any section. The parameters will then be fixed under the conservation of two moments of the distribution function. It is a slightly different approach compared to the classical multi-fluid model because in this approach, the corresponding $\kappa_{i}$ defined in Equation (1.7) is now a function also depending on $t$ and $x$.

\subsubsection{Determination of a function using its moments}

We begin by introducing some notations: Let $s$ be the surface variable (results can be stated for any size variable such as radius, volume ...), and $N$ be a positive integer. $\left(I_{i}\right)_{0 \leq i \leq N}$ will denote the set of sections and for any real $\alpha \geq 0, M_{i}^{\alpha}(f)$ will denote the moment of order $\alpha$ of $f$ on $I_{i}$ :

$$
M_{i}^{\alpha}(f)=\frac{1}{\left|I_{i}\right|} \int_{I_{i}} s^{\alpha} f(s) \mathrm{d} s .
$$

Having chosen a set $\left(I_{i}\right)_{i}$ of intervals and the orders of the moments $\left(\alpha_{j}\right)_{j}$, we can then define the following equivalence relation on $\mathcal{L}=\mathrm{L}^{1}\left(\mathbb{R}_{+},\left(\sum_{j} s^{\alpha_{j}}\right) \mathrm{d} s\right)$ :

$$
f \Re g \Leftrightarrow M_{i}^{\alpha_{j}}(f)=M_{i}^{\alpha_{j}}(g) \quad \forall i, j .
$$

We are interested in the space of functions $\mathcal{L} / \Re$. With such an approach, the difficulty is to find a family of functions $\mathcal{F}$ such that for any $f \in \mathcal{L}$, there exists one and only one $g \in \mathcal{F}$ satisfying $f \Re g$. This will be called the realizability condition.

\subsubsection{Exponential family and realizability condition}

In this paper we propose one kind of approximation relying on two moments of the density function. More precisely, we choose to preserve two moments in the surface variable: these are the 0 th and $3 / 2$ th order ones, which can be interpreted respectively as the number and mass densities of the distribution function. Knowing both quantities in any section enables us to have an information on the mean surface of the droplet distribution within any single section thanks to the mean formula:

$$
\bar{s}_{i}=\left(\frac{\int_{I_{i}} s^{3 / 2} f(s) \mathrm{d} s}{\int_{I_{i}} f(s) \mathrm{d} s}\right)^{\frac{2}{3}}=\left(\frac{M_{i}^{3 / 2}(f)}{M_{i}^{0}(f)}\right)^{\frac{2}{3}} .
$$

As stated in the introduction, one can remark that in the case where only one section is used, the corresponding multi-fluid model will correspond to a classical "two-fluid model" with three equations for the particulate phase: one for the number density, one for the mass density and one for the momentum (see for example $[13,21,34]$ ), the droplet mean size being given by formula (1.10).

The moments to be preserved being chosen, there still remains to find a well-suited family to represent the class $\mathcal{L} / \Re$. The following proposition gives a family of functions satisfying the realizability condition: 
Proposition 1.6. Let $I$ be any (finite or not) interval of $\left[0,+\infty\left[\right.\right.$ and $f \in \mathrm{L}^{1}\left(I,\left(1+s^{3 / 2}\right) \mathrm{d} s\right)$ be a positive function not everywhere equal to zero. Then there exists a unique couple $(a, b) \in \mathbb{R}_{+} \times \mathbb{R}$ such that the function defined by $\tilde{f}(s)=a \exp (-b s)$ has the same moments of order 0 and $3 / 2$ on $I$ as $f$. Moreover if the interval I is infinite we have $b>0$. We can then define a one-to-one function $\Psi$ such that $(a, b)=\Psi_{I}\left(M_{I}^{3 / 2}(f), M_{I}^{0}(f)\right)$.

Proof. For convenience, we will assume that our interval is finite and note $I=] s_{1}, s_{2}[, n$ and $m$ are respectively the moments of order 0 and $3 / 2$ of $f$. We will also note $\tilde{m}=\tilde{m}(a, b)$ and $\tilde{n}=\tilde{n}(a, b)$ the moments of order $3 / 2$ and 0 of the function $\tilde{f}(s)=a \exp (-b s)$. In order to simplify the notations, we will suppose that $\rho_{l} /(3 \sqrt{4 \pi})=1$.

Since $f$ is a positive function, we know that the ratio $m / n$ lies within the interval $] s_{1}^{3 / 2}, s_{2}^{3 / 2}[$. The ratio $\tilde{m} / \tilde{n}$ only depends on the parameter $b$, we will then note $g_{I}(b)$ this ratio. Let us first remark that if we have determined $b \neq 0$ such that $\tilde{m} / \tilde{n}=m / n$, it immediately follows that for:

$$
a=\frac{n b \Delta s}{\mathrm{e}^{-b s_{1}}-\mathrm{e}^{-b s_{2}}}
$$

we have both $n=\tilde{n}$ and $m=\tilde{m}$. Then we only have to check that $g_{I}$ as a function of $b \in \mathbb{R}$ is one-to-one onto ]$s_{1}^{3 / 2}, s_{2}^{3 / 2}$, which is the purpose of the following lemma. The whole process is exactly the same and presents no more difficulty in the case where the interval $I$ is infinite, which concludes the proof.

Lemma 1.7. The function $g_{I}$ defined in the preceding proof is strictly decreasing on $\mathbb{R}$ and has the following properties: $\lim _{b \rightarrow-\infty} g_{I}(b)=s_{2}^{3 / 2}$ and $\lim _{b \rightarrow+\infty} g_{I}(b)=s_{1}^{3 / 2}$.

Proof. For any $b \neq 0$ we have, denoting $\Delta s=s_{2}-s_{1}$ :

$$
g_{I}(b)=\frac{1}{1-\mathrm{e}^{-b \Delta s}}\left(s_{1}^{3 / 2}-s_{2}^{3 / 2} \mathrm{e}^{-b \Delta s}+\frac{3}{2 b}\left(\sqrt{s_{1}}-\sqrt{s_{2}} \mathrm{e}^{-b \Delta s}\right)+\frac{3 \mathrm{e}^{-b s_{1}}}{2 b} \int_{\sqrt{s_{1}}}^{\sqrt{s_{2}}} \mathrm{e}^{-b r^{2}} \mathrm{~d} r\right) .
$$

It follows easily that $\lim _{b \rightarrow-\infty} g_{I}(b)=s_{2}^{3 / 2}$ and $\lim _{b \rightarrow+\infty} g_{I}(b)=s_{1}^{3 / 2}$. We then know that $g_{I}$ is onto $] s_{1}^{3 / 2}, s_{2}^{3 / 2}[$. Moreover, $g_{I}$ is obviously $\mathcal{C}^{\infty}$ and we can compute $g_{I}^{\prime}$ in order to find its sign:

$$
g_{I}^{\prime}(b)=\frac{-\int_{s_{1}}^{s_{2}} \mathrm{e}^{-b s} \mathrm{~d} s \int_{s_{1}}^{s_{2}} s^{5 / 2} \mathrm{e}^{-b s} \mathrm{~d} s+\int_{s_{1}}^{s_{1}} s^{3 / 2} \mathrm{e}^{-b s} \mathrm{~d} s \int_{s_{1}}^{s_{2}} s \mathrm{e}^{-b s} \mathrm{~d} s}{\left(\int_{s_{1}}^{s_{2}} \mathrm{e}^{-b s} \mathrm{~d} s\right)^{2}} .
$$

Let us keep $b$ fixed and determine the sign of:

$$
h(\beta)=-\int_{s_{1}}^{\beta} \mathrm{e}^{-b s} \mathrm{~d} s \int_{s_{1}}^{\beta} s^{5 / 2} \mathrm{e}^{-b s} \mathrm{~d} s+\int_{s_{1}}^{\beta} s^{3 / 2} \mathrm{e}^{-b s} \mathrm{~d} s \int_{s_{1}}^{\beta} s \mathrm{e}^{-b s} \mathrm{~d} s .
$$

The computation of the derivative yields:

$$
\begin{gathered}
h^{\prime}(\beta)=\mathrm{e}^{-b \beta} \int_{s_{1}}^{\beta}\left(-s^{5 / 2}-\beta^{5 / 2}+\beta^{3 / 2} s+\beta s^{3 / 2}\right) \mathrm{e}^{-b s} \mathrm{~d} s, \\
h^{\prime}(\beta)=\mathrm{e}^{-b \beta} \int_{s_{1}}^{\beta}\left(\beta^{3 / 2}-s^{3 / 2}\right)(s-\beta) \mathrm{e}^{-b s} \mathrm{~d} s .
\end{gathered}
$$

We can now state that $h^{\prime}(\beta)<0$ if $\beta>s_{1}$ and as we have $h\left(s_{1}\right)=0$, we also have $g_{I}^{\prime}(b)<0$ for any $b \in \mathbb{R}$. Then $g_{I}$ is strictly decreasing on $\mathbb{R}$. 
Let us then define:

$$
\mathcal{F}=\left\{s \mapsto \sum_{i=1}^{N} \mathbb{I}_{s_{i-1} \leq s<s_{i}} a_{i} \exp \left(-b_{i} s\right)+\mathbb{I}_{s>s_{N}} a_{N+1} \exp \left(-b_{N+1} s\right) /\left(a_{i}, b_{i}\right) \in \mathbb{R}_{+} \times \mathbb{R} \forall i \text { and } b_{N+1}>0\right\}
$$

The last result shows that this exponential family $\mathcal{F}$ fulfils the realizability condition. Moreover choosing such a family of functions has two more advantages: the first is that the positivity of the number density (which is not obvious for affine functions) is always preserved and the second is that we have the possibility to use only one section (which is equivalent to take $N=0$ ), obtaining a two-fluid-type model. This result also allows us to define a nonlinear projection operator $\Pi_{I}: L^{1}\left(I,\left(1+s^{3 / 2}\right) \mathrm{d} s\right) \mapsto \mathcal{F}$.

Remark 1.8. Concerning the evaporation phenomenon, the choice of an exponential function is well-suited since such kinds of distributions are proved to be self-similar during their evolution in time. Some remarks can also be found in [26] when the choice of a distribution function for the last section (the infinite one) is discussed.

\subsubsection{Second order multi-fluid model}

We recall that $\left(a_{i}, b_{i}\right)=\Psi_{\left[s_{i-1}, s_{i}[\right.}\left(m_{i}, n_{i}\right)$ where $\Psi_{\left[s_{i-1}, s_{i}[\right.}$ is defined in Proposition 1.6. Following the same process as for the classical multi-fluid method, we consider the system (1.8) and thanks to Proposition 1.6, we assume that in any section, the quantity $n(t, x, s)$ is given by $n(t, x, s)=a_{i}(t, x) \exp \left(-b_{i}(t, x) s\right)$. In order to clarify the notations, the $(t, x)$ dependence of $a_{i}$ and $b_{i}$ will be omitted in the following.

The classical multi-fluid model consists in deriving evolution equations for two quantities in any section which are mass density and momentum. Since we have chosen to preserve two moments in the surface variable we have to deduce evolution equations on three quantities in any section: the number density $n_{i}$, the mass density $m_{i}$ and the momentum density $m_{i} \bar{v}_{i}$, where

$$
n_{i}(t, x)=\frac{1}{s_{i}-s_{i-1}} \int_{s_{i-1}}^{s_{i}} a_{i} \exp \left(-b_{i} s\right) \mathrm{d} s \quad ; \quad m_{i}(t, x)=\frac{1}{s_{i}-s_{i-1}} \int_{s_{i-1}}^{s_{i}} \frac{\rho_{l} s^{3 / 2}}{3 \sqrt{4 \pi}} a_{i} \exp \left(-b_{i} s\right) \mathrm{d} s .
$$

To derive these equations, in any section, multiply the first equation of the system (1.8) respectively by 1 and $\frac{\rho_{l} s^{3 / 2}}{3 \sqrt{4 \pi}}$ and the second equation by $\frac{\rho_{l} s^{3 / 2}}{3 \sqrt{4 \pi}}$ and integrate the resulting three equations over the whole section, recalling the particular form for $n_{i}$ and $m_{i}$ (1.13). The improved sectional approach then consists in a set of $3 N+3$ equations defined for $1 \leq i \leq N+1$ :

$$
\left\{\begin{aligned}
\partial_{t} n_{i}+\partial_{x}\left(n_{i} \bar{v}_{i}\right)+K . F_{i}-K F_{i+1} & =0 \\
\partial_{t} m_{i}+\partial_{x}\left(m_{i} \bar{v}_{i}\right)+\left(E_{i}^{(1)}+E_{i}^{(2)}\right) m_{i}-E_{i+1}^{(1)} m_{i+1} & =0 \\
\partial_{t}\left(m_{i} \bar{v}_{i}\right)+\partial_{x}\left(m_{i} \bar{v}_{i} \otimes \bar{v}_{i}\right)+\left(E_{i}^{(1)}+E_{i}^{(2)}\right) m_{i} \bar{v}_{i}-E_{i+1}^{(1)} m_{i+1} \bar{v}_{i+1} & =m_{i} \bar{A}_{i}
\end{aligned}\right.
$$

where

$$
\begin{gathered}
E_{i}^{(1)}=\frac{\rho_{l} K}{3 \sqrt{4 \pi}} s_{i-1}^{3 / 2} a_{i} \exp \left(-b_{i} s_{i-1}\right), \quad E_{i}^{(2)}=\int_{s_{i-1}}^{s_{i}} \frac{\rho_{l} K}{3 \sqrt{4 \pi}} s^{1 / 2} a_{i} \exp \left(-b_{i} s\right) \mathrm{d} s \\
\overline{A_{i}}=\int_{s_{i-1}}^{s_{i}} \frac{\rho_{l} s^{3 / 2}}{3 \sqrt{4 \pi}} A\left(t, x, s, \bar{v}_{i}\right) a_{i} \exp \left(-b_{i} s\right) \mathrm{d} s, \quad F_{i}=a_{i} \exp \left(-b_{i} s_{i-1}\right)
\end{gathered}
$$

with the convention that $E_{N+2}=0$ and $s_{N+1}=+\infty$. We emphasize once more the fact that $a_{i}$ and $b_{i}$ are both functions depending only on $m_{i}$ and $n_{i}$ so that the model is closed. In order to avoid long notations, in all the following, the "exponential model" will denote the second-order multi-fluid model obtained using the exponential approximation. 


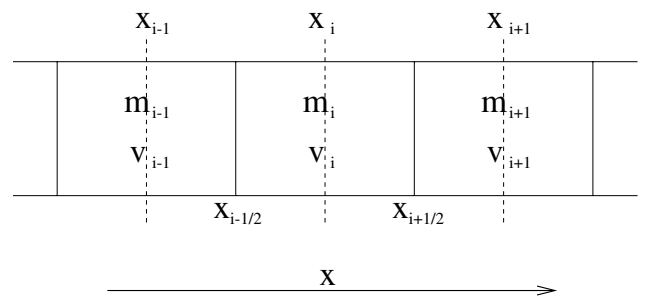

FiguRE 1. Discretization for the transport part.

\section{The NUMERICAL SCHEMES}

\subsection{Time discretization}

In this section we present the numerical scheme we have developed in order to solve the system (1.14). Since the numerical methods used for the transport part (finite volume method) and for the evaporation part (improved sectional method) follow different approaches, these two effects are computed separately, by using a fractional step in time method in order to solve the global system. The Strang splitting method ensures us to have a second order accurate method for the time discretization if both schemes for transport and evaporation are second order accurate. Such a splitting is done in the following way: if $S_{\Delta t}, T_{\Delta t}$ and $E_{\Delta t}$ denote respectively the schemes for the Strang method, and the transport and evaporation steps

$$
S_{\Delta t}=E_{\Delta t / 2} \circ T_{\Delta t} \circ E_{\Delta t / 2}
$$

The reader may refer for example to [36] or [5] for further details. The second-order accuracy in time for any scheme will be ensured using a classical RK2 method.

\subsection{The transport scheme}

Concerning the scheme used for the convection part, we first have to emphasize that there is one constraint on $m$ and $n$ that must be satisfied. Indeed, in any section $i$, since $n_{i}$ and $m_{i}$ denote respectively the number and mass density in the section, their ratio $m_{i} / n_{i}$ has therefore to remain in the following interval: $] \frac{\rho_{L}}{3 \sqrt{4 \pi}} s_{i-1}^{3 / 2}, \frac{\rho_{L}}{3 \sqrt{4 \pi}} s_{i}^{3 / 2}$ [.

Thus the transport scheme has to preserve this property on $n_{i}$ and $m_{i}$. The section being fixed, we will omit to note the index $i$ related to the size and note $\mu=m / n$ the mean mass of a droplet a section so that we are solving:

$$
\left\{\begin{aligned}
\partial_{t} n+\nabla_{x} \cdot(n v) & =0 \\
\partial_{t}(n \mu)+\nabla_{x} \cdot(n \mu v) & =0 \\
\partial_{t}(n \mu v)+\nabla_{x} \cdot(n \mu v \otimes v) & =0 .
\end{aligned}\right.
$$

Remark 2.1. The system (2.1) (transport part) is closely related to the "pressureless gas dynamics system". It is well-known that solutions to the pressureless gases system may have a rather complicated behaviour since, even for regular initial condition, mass concentration as well as vacuum may appear in a finite time lap. This system has been widely studied and is related to the problem of solving linear transport equation with nonsmooth coefficients (see for example $[6,7,10,31]$ ).

\subsubsection{First-order scheme}

We begin by studying the first order numerical scheme for transport in one dimension of space. We will here use conservative variables $n, m$ and $p$, defining the velocity $v$ as $v=p / m$. It is quite obvious to see that the pressureless gas dynamics system is not hyperbolic. We first turn this system into an hyperbolic one by adding a pressure term, which is equivalent to assume that there is small dispersion of the droplets velocities at the microscopic level and that the term $\nabla_{x} . P$ defined in (1.4) is non-zero. The system (2.1) may be seen as the 
limit as $\epsilon$ tends toward zero of the following hyperbolic system:

$$
\left\{\begin{aligned}
\partial_{t} n+\nabla_{x} \cdot(n v) & =0 \\
\partial_{t} m+\nabla_{x} \cdot(m v) & =0 \\
\partial_{t}(m v)+\nabla_{x} \cdot(m v \otimes v)+\epsilon^{2} \partial_{x} m & =0 .
\end{aligned}\right.
$$

As we are in one space dimension, one may derive a classical Roe-Scheme for $\epsilon>0$ and then let $\epsilon$ vanish in the resulting scheme [14]. The final scheme reads, with $\lambda_{i}=\Delta t / \Delta x_{i}$ and $\Delta x_{i}=x_{i+1 / 2}-x_{i-1 / 2}$ :

$$
\left\{\begin{array}{l}
n_{i}^{k+1}=n_{i}^{k}+\frac{\lambda_{i}}{2}\left(n_{i+1}^{k}\left(\left|v_{i+1}^{k}\right|-v_{i+1}^{k}\right)-2 n_{i}^{k}\left|v_{i}^{k}\right|+n_{i-1}^{k}\left(\left|v_{i-1}^{k}\right|+v_{i-1}^{k}\right)\right) \\
m_{i}^{k+1}=m_{i}^{k}+\frac{\lambda_{i}}{2}\left(m_{i+1}^{k}\left(\left|v_{i+1}^{k}\right|-v_{i+1}^{k}\right)-2 m_{i}^{k}\left|v_{i}^{k}\right|+m_{i-1}^{k}\left(\left|v_{i-1}^{k}\right|+v_{i-1}^{k}\right)\right) \\
p_{i}^{k+1}=p_{i}^{k}+\frac{\lambda_{i}}{2}\left(p_{i+1}^{k}\left(\left|v_{i+1}^{k}\right|-v_{i+1}^{k}\right)-2 p_{i}^{k}\left|v_{i}^{k}\right|+p_{i-1}^{k}\left(\left|v_{i-1}^{k}\right|+v_{i-1}^{k}\right)\right) .
\end{array}\right.
$$

Proposition 2.2. Under the CFL condition $\max _{i} \lambda_{i}\left|v_{i}\right|<1$, this scheme preserves the positivity of both mass and number density and moreover we have a maximum principle on $\mu=\frac{m}{n}$ and $v$.

Proof. Rewriting the numerical scheme (2.2) on the number density as:

$$
n_{i}^{k+1}=n_{i}^{k}\left(1-\lambda_{i}\left|v_{i}^{k}\right|\right)+\frac{\lambda_{i}}{2}\left(n_{i+1}^{k}\left(\left|v_{i+1}^{k}\right|-v_{i+1}^{k}\right)+n_{i-1}^{k}\left(\left|v_{i-1}^{k}\right|+v_{i-1}^{k}\right)\right)
$$

and remarking that $(|\alpha| \pm \alpha) \geq 0$ for any $\alpha \in \mathbb{R}$, we easily have the positivity for the number density and the mass density is treated the same way. Let us now write the numerical scheme on the mass density as:

$$
n_{i}^{k+1} \mu_{i}^{k+1}=n_{i}^{k} \mu_{i}^{k}\left(1-\lambda_{i}\left|v_{i}^{k}\right|\right)+\frac{\lambda_{i}}{2}\left(n_{i+1}^{k} \mu_{i}^{k}\left(\left|v_{i+1}^{k}\right|-v_{i+1}^{k}\right)+n_{i-1}^{k} \mu_{i}^{k}\left(\left|v_{i-1}^{k}\right|+v_{i-1}^{k}\right)\right) .
$$

Using the fact that the number density remains positive, we see that $\mu_{i}^{k+1}$ is a convex combination of $\mu_{i-1}^{k}, \mu_{i}^{k}$ and $\mu_{i+1}^{k}$. The same argument apply to the velocity and this concludes the proof.

Remark 2.3. The scheme (2.2) may be written in conservative form:

$$
\left(\begin{array}{c}
n_{i}^{k+1} \\
m_{i}^{k+1} \\
p_{i}^{k+1}
\end{array}\right)=\left(\begin{array}{c}
n_{i}^{k} \\
m_{i}^{k} \\
p_{i}^{k}
\end{array}\right)-\lambda\left(\begin{array}{l}
F_{i+1 / 2}^{k,(1)}-F_{i-1 / 2}^{k,(1)} \\
F_{i+1 / 2}^{k,(2)}-F_{i-1 / 2}^{k,(2)} \\
F_{i+1 / 2}^{k,(3)}-F_{i-1 / 2}^{k,(3)}
\end{array}\right)
$$

where the numerical fluxes $F_{i+1 / 2}^{k}$ is defined as:

$$
\left\{\begin{array}{l}
F_{i+1 / 2}^{k,(1)}=n_{i+1}^{k} \min \left(0, v_{i+1}^{k}\right)+n_{i}^{k} \max \left(0, v_{i}^{k}\right) \\
F_{i+1 / 2}^{k,(2)}=\mu_{i+1}^{k} \min \left(0, F_{i+1 / 2}^{k,(1)}\right)+\mu_{i}^{k} \max \left(0, F_{i+1 / 2}^{k,(1)}\right) \\
F_{i+1 / 2}^{k,(3)}=v_{i+1}^{k} \min \left(0, F_{i+1 / 2}^{k,(2)}\right)+v_{i}^{k} \max \left(0, F_{i+1 / 2}^{k,(2)}\right) .
\end{array}\right.
$$

Remark 2.4. The scheme (2.2) is the same as the first order kinetic scheme obtained by Bouchut et al. in [7] for the pressureless gas dynamics system. Using a technique of affine reconstruction and slope limitation, they also provided a second-order scheme verifying the preservation of the positivity and a maximum principle on the velocity. The scheme obtained by Bouchut et al. may easily be extended to higher dimensions, but it requires 
a cartesian grid for discretization. In order to remain as general as possible for the space discretization, we choose to derive a different second-order mesh-independant scheme which may be seen in higher dimensions as a convex combination of the one-dimensional scheme defined in (2.2).

\subsubsection{Second-order scheme}

We now focus on the description of the second-order scheme. As the numerical simulations presented at the end of the paper deal with one space dimension, the scheme will only be presented in a one-dimensional context. However, this scheme can easily be extended in higher dimensional cases as it is explained in Remark 2.5.

We recall the notations defined in Figure 1. The second order accuracy of the scheme in time and space can be ensured using both MUSCL-type and Runge-Kutta methods. Let $U^{k}$ be the values of the state $U=(n, m, v)^{T}$ at time $t^{k}$. An approximation of the gradient of the discrete solution in any cell is first computed in order to define interpolated values on all edges of the mesh. In order to avoid the creation of new extrema in the rebuild solution, we choose to follow the classical way in limiting the gradient. But since the quantities we want to control are $n, \mu$ and $v$, the limiting procedure is performed on the vector $W=(n, \mu, v)^{T}$ rather than on the vector $U$. With $W_{i}^{k}$ being the values of the state $W$ in the cell $C_{i}=\left[x_{i-1 / 2}, x_{i+1 / 2}\right.$ [at time $t^{k}$, an approximation of the gradient of the discrete solution is given by the formula:

$$
\nabla W_{i}^{k}=\frac{2\left(W_{i+1}^{k}-W_{i-1}^{k}\right)}{\Delta x_{i-1}+2 \Delta x_{i}+\Delta x_{i+1}} .
$$

As for the limitation part, we have to replace $\nabla W_{i}^{k}$ with its limited value $\tilde{\nabla} W_{i}^{k}$ in each cell $C_{i}$. An appropriate way of limiting the gradient (see Prop. 3.1) reads:

$$
\tilde{\nabla} W_{i}^{k}=\min \left(1, \frac{2\left(W_{\max , i}^{k}-W_{i}^{k}\right)}{\left|\nabla W_{i}^{k}\right| \cdot \Delta x_{i}}, \frac{2\left(W_{i}^{k}-W_{\min , i}^{k}\right)}{\left|\nabla W_{i}^{k}\right| \cdot \Delta x_{i}}\right) \cdot \nabla W_{i}^{k}
$$

where $W_{\max , i}^{k}=\max _{j \in\{i-1, i, i+1\}}\left(W_{j}^{k}\right)$ and $W_{\min , i}=\min _{j \in\{i-1, i, i+1\}}\left(W_{j}^{k}\right)$.

Then, knowing the value of the gradient we can now give interpolated values of $W^{k}$ at the edges of the mesh and define the values used for the computation of the fluxes.

$$
\begin{aligned}
& W_{i, l}^{k}=W_{i}^{k}-\tilde{\nabla} W_{i}^{k} \cdot \frac{\Delta x_{i}}{2} \\
& W_{i, r}^{k}=W_{i}^{k}+\tilde{\nabla} W_{i}^{k} \cdot \frac{\Delta x_{i}}{2} .
\end{aligned}
$$

With the notations defined in the previous part, the numerical fluxes can be written:

$$
\left\{\begin{array}{l}
F_{i+1 / 2}^{k,(1)}=W_{i, r}^{k,(1)} \min \left(0, W_{i, r}^{k,(3)}\right)+W_{i, l}^{k,(1)} \max \left(0, W_{i, l}^{k,(3)}\right) \\
F_{i+1 / 2}^{k,(2)}=W_{i, r}^{k,(2)} \min \left(0, F_{i+1 / 2}^{k,(1)}\right)+W_{i, l}^{k,(2)} \max \left(0, F_{i+1 / 2}^{k,(1)}\right) \\
F_{i+1 / 2}^{k,(3)}=W_{i, r}^{k,(3)} \min \left(0, F_{i+1 / 2}^{k,(2)}\right)+W_{i, l}^{k,(3)} \max \left(0, F_{i+1 / 2}^{k,(2)}\right) .
\end{array}\right.
$$

Finally, the values of $U^{k+1}$ at time $t_{k+1}$ are defined using a Runge-Kutta method ensuring that the positivity is preserved (see Prop. 3.1): we first compute $\tilde{U}_{i}^{k+1}$ using the following formula:

$$
\tilde{U}_{i}^{k+1}=U_{i}^{k}-\frac{\Delta t}{\Delta x_{i}}\left(F_{i+1 / 2}^{k}-F_{i-1 / 2}^{k}\right)
$$

and, with obvious notations, we can compute $U_{i}^{k+1}$ by:

$$
U_{i}^{k+1}=\frac{1}{2} U_{i}^{k}+\frac{1}{2} \tilde{U}_{i}^{k+1}-\frac{\Delta t}{2 \Delta x_{i}}\left(\tilde{F}_{i+1 / 2}^{k}-\tilde{F}_{i-1 / 2}^{k}\right) .
$$


Remark 2.5. The scheme we just described can easily be extended in higher dimension. Indeed, one can remark that an extension to formula (2.4) can directly be provided using the Green formula applied to the dual mesh (see for example [14]). The same kind of limiters may then be used, provided that the two terms $\left|\nabla W_{i}^{k}\right| . \Delta x_{i} / 2$ are respectively replaced with $\max _{x \in \partial C_{i}}\left|\overrightarrow{\nabla W_{i}^{k}} \cdot\left(x-x_{i}\right)\right|$ and $\min _{x \in \partial C_{i}}\left|\overrightarrow{\nabla W_{i}^{k}} \cdot\left(x-x_{i}\right)\right|$. The expression for the multidimensional scheme, equivalent to formula (2.9) (i.e. without the Runge-Kutta time integration) then reads:

$$
U_{i}^{k+1}=U_{i}^{k}-\frac{\Delta t}{\left|C_{i}\right|} \sum_{e \in \partial C_{i}} \Phi\left(U_{i}^{k}, U_{i, e}^{k}, \overrightarrow{n_{i, e}}\right)|e|
$$

where $\Phi\left(U_{i}^{k}, U_{i, e}^{k}, \overrightarrow{n_{i, e}}\right)$ is the numerical flux through the edge $e$ of the boundary of the cell $C_{i}$ and is defined as

$$
\Phi\left(U_{1}, U_{2}, \overrightarrow{n_{1, e}}\right)=U_{1} \max \left(0, \overrightarrow{v_{1}} \cdot \overrightarrow{n_{1, e}}\right)+U_{2} \min \left(0, \overrightarrow{v_{2}} \cdot \overrightarrow{n_{1, e}}\right)
$$

This formula can then be understood as a convex combination of the monodimensional formula and the results from Proposition 3.1 remain valid assuming that an adapted CFL condition is used [14].

\subsection{Computation of the drag force effects}

The drag force effect can either be treated as a source term in the transport part or as a separated scheme using one more time the Strang splitting with an exact integration (i.e. replace $T_{\Delta t}$ with $D_{\Delta t / 2} \circ T_{\Delta t} \circ D_{\Delta t / 2}$ ). Since the drag source term may become stiff for small droplets, the second possibility has been chosen. We recall the expression of the drag force term following a Stokes law, where $s$ denotes the surface of the droplets:

$$
A(t, x, v, s)=\frac{U_{g}(t, x)-v}{\gamma s}
$$

where $\gamma=\frac{\rho_{l}}{18 \pi \mu_{g}}$. In the sectional approach, the value of the drag force term in the $i$ th section reads:

$$
\bar{A}_{i}(t, x, s)=\frac{U_{g}(t, x)-\bar{v}_{i}(t, x)}{\gamma s} .
$$

The part of the system we are solving then reads in any section $1 \leq i \leq N+1$ :

$$
\left\{\begin{aligned}
\partial_{t} m_{i} & =0 \\
\partial_{t}\left(m_{i} \bar{v}_{i}\right) & =\int_{s_{i-1}}^{s_{i}} \frac{\rho_{l} s^{3 / 2}}{3 \sqrt{4 \pi}} a_{i} \exp \left(-b_{i} s\right) \bar{A}_{i}(t, x, s) \mathrm{d} s .
\end{aligned}\right.
$$

In any section, we are looking for a mean value in order to represent the time it takes for a droplet to adapt to the velocity of the gas. This mean value is defined through the following integral:

$$
\frac{m_{i}}{\tau_{i}}=\int_{s_{i-1}}^{s_{i}} \frac{\rho_{l} s^{3 / 2}}{3 \sqrt{4 \pi}} \frac{a_{i} \exp \left(-b_{i} s\right)}{\gamma s} \mathrm{~d} s .
$$

After some algebra, we obtain in any section with the convention $s_{N+1}=+\infty$ :

$$
\tau_{i}=\frac{3 \gamma m_{i}}{2\left(a_{i}\left[s^{3 / 2} \exp \left(-b_{i} s\right)\right]_{s_{i-1}}^{s_{i}}+b_{i} m_{i}\right)} .
$$

Since the drag force does not have any effect on the mass contained in the section but only on the momentum, the system (2.13) can be simplified, removing the dependence on the mass density and yielding the value of the 
velocity after one time step:

$$
\bar{v}_{i}\left(t^{k+1}\right)=U_{g}+\left(\bar{v}_{i}\left(t^{k}\right)-U_{g}\right) \exp \left(-\frac{\Delta t}{\tau_{i}}\right)
$$

This scheme needs no CFL condition to preserve the maximum principle on the velocity, whereas using source terms would require a CFL shrinking to zero as the size of the droplets goes to zero.

\subsection{Numerical scheme for evaporation}

In this paragraph, focus is set upon the evaporation step, using the kinetic origin of the system (1.14) to derive a numerical scheme. Omitting all terms excepted the evaporation one, the kinetic equation written in the surface variable $s$ reads:

$$
\partial_{t} f-K \partial_{s} f=0
$$

where $K$ is assumed to be a constant. Given an initial condition $f_{0}$, the exact solution of this equation for any time $t>0$ reads: $f(t, s)=f_{0}(s+K t)$, for all $(t, s) \geq 0$.

Let $\left(s_{i}\right)_{0 \leq i \leq N}$ be a discretization of the size space in the surface variable. A time step for the evaporation scheme may be decomposed into three elementary steps. The first one consists in rebuilding the approximate number density function knowing the number and the mass density in every section (projection step $\Pi_{\text {exp }}$ ). More precisely we compute the only element $\tilde{f}$ of the family $\mathcal{F}$ (defined in (1.12)) which has the appropriate moments of order 0 and $3 / 2$ in any section. Then the second step consists in using the exact solution of Equation (2.16) in order to transport exactly the rebuilt density (transport step $T_{\Delta t}$ ). Finally the new number and mass density are computed in every section (mean step $\Pi_{\text {mean }}$ ). If $p$ is related to the time discretization and $i$ is related to the size discretization we have:

$$
\left(\begin{array}{c}
n_{i}^{p+1} \\
m_{i}^{p+1} \\
\left(m_{i} \bar{v}_{i}\right)^{p+1}
\end{array}\right)_{i}=\Pi_{\text {mean }} \circ T_{\Delta t} \circ \Pi_{\exp }\left(\begin{array}{c}
n_{i}^{p} \\
m_{i}^{p} \\
\left(m_{i} \bar{v}_{i}\right)^{p}
\end{array}\right)_{i} .
$$

The scheme can be written under its "conservative" form where $\Delta s_{i}=s_{i+1}-s_{i}$ :

$$
\left\{\begin{aligned}
\frac{n_{i}^{p+1}-n_{i}^{p}}{\Delta t}-K \frac{\varphi_{i+1 / 2}^{p+1 / 2}-\varphi_{i-/ 2}^{p+1 / 2}}{\Delta s_{i}} & =0 \\
\frac{m_{i}^{p+1}-m_{i}^{p}}{\Delta t}-K \frac{\psi_{i+1 / 2}^{p+1 / 2}-\psi_{i-/ 2}^{p+1 / 2}}{\Delta s_{i}} & =S_{i} \\
\frac{m_{i}^{p+1} \bar{v}_{i}^{p+1}-m_{i}^{p} \bar{v}_{i}^{p}}{\Delta t}-K \frac{\theta_{i+1 / 2}^{p+1 / 2}-\theta_{i-1 / 2}^{p+1 / 2}}{\Delta s_{i}} & =T_{i}
\end{aligned}\right.
$$

and where $\varphi, \psi, \theta, S$ and $T$ are defined as follows:

$$
\begin{aligned}
\varphi_{i+1 / 2}^{p+1 / 2} & =\frac{1}{\Delta t} \int_{0}^{\Delta t} \tilde{f}\left(s_{i+1}+K t\right) \mathrm{d} t \\
\psi_{i+1 / 2}^{p+1 / 2} & =\frac{1}{\Delta t} \int_{0}^{\Delta t}\left(s_{i+1}+K t\right)^{3 / 2} \tilde{f}\left(s_{i+1}+K t\right) \mathrm{d} t \\
\theta_{i+1 / 2}^{p+1 / 2} & =\frac{1}{\Delta t} \int_{0}^{\Delta t}\left(s_{i+1}+K t\right)^{3 / 2} \tilde{f}\left(s_{i+1}+K t\right) \bar{v}_{i+1} \mathrm{~d} t \\
S_{i} & =\frac{1}{\Delta s_{i} \Delta t} \int_{0}^{\Delta s_{i}}\left(\left(s_{i}+u\right)^{3 / 2}-\left(s_{i}+u+K \Delta t\right)^{3 / 2}\right) \tilde{f}\left(s_{i}+K \Delta t+u\right) \mathrm{d} u \\
T_{i} & =\frac{1}{\Delta s_{i} \Delta t} \int_{0}^{\Delta s_{i}}\left(\left(s_{i}+u\right)^{3 / 2}-\left(s_{i}+u+K \Delta t\right)^{3 / 2}\right) \tilde{f}\left(s_{i}+K \Delta t+u\right) \bar{v}\left(s_{i}+u+K \Delta t\right) \mathrm{d} u .
\end{aligned}
$$


We recall that the notation $\tilde{f}$ denotes the global density function which is rebuilt from the number and the mass given in any section. Using some integrals of the function $s^{3 / 2} \exp (-b s)$ which can be computed numerically and denoting $\alpha_{i}=K \Delta t / \Delta s_{i}$, this scheme may also be written the following way:

$$
\begin{aligned}
n_{i}^{p+1}= & n_{i}^{p}\left(1-g\left(b_{i} K \Delta t, \alpha_{i}\right)\right)+n_{i+1}^{p} g\left(b_{i+1} K \Delta t, \alpha_{i+1}\right) \\
m_{i}^{p+1}= & n_{i}^{p} \frac{b_{i} \exp \left(-b_{i} K \Delta t\right)}{1-\exp \left(-b_{i} \Delta s_{i}\right)} \int_{0}^{\Delta s_{i}-K \Delta t}\left(s+s_{i}\right)^{3 / 2} \exp \left(-b_{i} s\right) \mathrm{d} s \\
& +n_{i+1}^{p} \frac{b_{i+1} \exp \left(-b_{i+1} s_{i}\right)}{1-\exp \left(-b_{i+1} \Delta s_{i+1}\right)} \int_{0}^{K \Delta t}\left(s+s_{i+1}-K \Delta t\right)^{3 / 2} \exp \left(-b_{i+1} s\right) \mathrm{d} s
\end{aligned}
$$

where the functions $g$ is defined as:

$$
g(x, \alpha)=\frac{1-\exp (-x)}{1-\exp (-\alpha x)} .
$$

Remark 2.6. Since $\alpha$ is the inverse of the CFL constant, it is assumed to be positive and more precisely to be greater than 1. Under this assumption we have for any $x \in \mathbb{R}: 0 \leq g(x, \alpha) \leq 1$. This ensures the positivity of the number density and therefore the positivity of the mass density. Moreover, the function $g$ is shown to have a Lipschitz dependence on the first variable (the one involving the time step) provided that the CFL condition is kept constant. Indeed, we can compute $\partial_{x} g$ :

$$
\partial_{x} g(x, \alpha)=\frac{\mathrm{e}^{-x}\left(1-\mathrm{e}^{-\alpha x}\right)-\left(1-\mathrm{e}^{-x}\right) \alpha \mathrm{e}^{-\alpha x}}{\left(1-\mathrm{e}^{-\alpha x}\right)^{2}} .
$$

Keeping $\alpha \geq 1$ fixed, we have $\lim _{x \rightarrow-\infty} \partial_{x} g(x, \alpha)=\lim _{x \rightarrow+\infty} \partial_{x} g(x, \alpha)=0$ and we also note that $\partial_{x} g(x, \alpha)$ admits a limit as $x \rightarrow 0$ :

$$
\partial_{x} g(0, \alpha)=\frac{\alpha-1}{2 \alpha}
$$

We deduce from this last equality that $\left|\partial_{x} g(., \alpha)\right|$ is bounded by a constant $M_{\alpha}$. This property will be used later in order to prove some properties of the scheme (2.23) (see Prop. 3.11).

\section{TheORETICAL RESUlts ON THE SCHEMES}

\subsection{General results}

We give here some general results on the schemes defined in the preceding part. The proofs of these results do not present any particular difficulty and we will thus just give the main lines. Concerning the part on the transport scheme, the reader may also refer to [7] for similar arguments.

Proposition 3.1. Under the CFL-condition $2 \Delta t \leq \Delta x$, using the second order transport scheme (2.10) gives a nonnegative number density and therefore a nonnegative mass density. Moreover, the velocity and the ratio $\mu=m / n$ do satisfy a maximum principle.

Proof. We have seen in Proposition 2.2 that the result holds for the one-dimensional first-order scheme. Let us now recall the notations from Section 2.2. The limiter defined in formula (2.5) provides an estimation of the values at the edges $W_{i, l}$ and $W_{i, r}$ compared to $W_{i}$, more precisely, we have $\left|W_{i, l}\right|,\left|W_{i, r}\right| \leq 2$. $\left|W_{i}\right|$ for any cell $C_{i}$. Then, recalling the fact that the first-order scheme does preserve the positivity of the number density with the CFL constant 1, the scheme (2.9) does also preserve the positivity under the CFL constant $1 / 2$.

The end of the proof now relies on a convexity argument. Indeed, the scheme (2.9) concerning the evolution of $\mu_{i}$ may be seen as a convex combination of the first-order scheme (2.2), which verifies the maximum principle on $\mu$. Then, the scheme (2.9) does also verify this maximum principle, ensuring the positivity of the mass density. The same argument holds for the maximum principle on the velocity. Finally, the limited gradient method for the transport scheme $(2.10)$ can also be seen as a convex combination between the state $U_{i}^{k}$ and the 
state $\tilde{U}_{i}^{k}$ since it is obtained using the scheme (2.9) applied twice respectively to $U_{i}^{k}$ and to $\tilde{U}_{i}^{k}$, which concludes the proof.

Proposition 3.2. The scheme simulating the drag force effects does preserve a modified maximum principle, including the velocity of the gas:

$$
\sup _{i}\left(\left|v_{i}^{p+1}\right|\right) \leq \max \left(\sup _{x}\left|U_{g}(x)\right|, \sup _{i}\left(\left|v_{i}^{p}\right|\right)\right)
$$

Proof. From formula (2.15) we have, for all $i, v_{i}^{p+1} \in\left[U_{g}, v_{i}^{p}\right]$.

Proposition 3.3. Under a $C F L$ condition $\Delta t \leq K \min _{i} \Delta s_{i}$, the evaporation scheme (2.17) does preserve the positivity of both number and mass density and also verifies a maximum principle on the velocity. Moreover, by construction of the scheme, in any section the ratio $m_{i} / n_{i}$ lies within the interval $] \frac{\rho_{L}}{3 \sqrt{4 \pi}} s_{i-1}^{3 / 2}, \frac{\rho_{L}}{3 \sqrt{4 \pi}} s_{i}^{3 / 2}[$.

Proof. Replacing respectively $n_{i}^{p}$ and $m_{i}^{p}$ with $\frac{1}{\Delta s_{i}} \int_{0}^{\Delta s_{i}}[\tilde{f}]\left(s_{i}+u\right) \mathrm{d} u$ and $\frac{1}{\Delta s_{i}} \int_{0}^{\Delta s_{i}}\left(s_{i}+u\right)^{3 / 2}[\tilde{f}]\left(s_{i}+u\right) \mathrm{d} u$ in the evaporation scheme (2.17), we see that $n_{i}^{p+1}$ and $m_{i}^{p+1}$ are obtained as number and mass densities corresponding to a piecewise exponential positive distribution function. We have then the positivity of both number and mass densities and the results on their ratio and on the velocity comes one more time from a convexity argument.

\subsection{Consistency of the evaporation scheme}

\subsubsection{Order of the exponential approximation}

Since it has been proven in Proposition 1.6 that the exponential family $\mathcal{F}$ fulfills the realizability condition, we will now focus on the order of the approximation achieved. Given an interval $I$ of length $h$, let $\Pi_{I}$ denote the projection operator which associates to a function $f \in \mathrm{L}^{1}\left(I,\left(1+s^{3 / 2}\right) \mathrm{d} s\right)$, the unique exponential function defined in Proposition 1.6. The following theorem gives explicit bounds for the error generated by replacing $f$ with $\Pi_{I}(f)$. In order to avoid heavy notations, the results in Theorem 3.4 and Lemma 3.5 are presented in a particular case. All results can be extended to a more general case without any difficulty, see Remark 3.7.

Theorem 3.4. Let $I$ be any interval of length $h$ and $s_{0}$ be its middle point. Let $f$ non identically equal to 0 be a function of class $\mathrm{C}^{3}$ such that $f\left(s_{0}\right) \neq 0$. We then have the following estimate:

$$
\left\|\Pi_{I}(f)-f\right\|_{\mathrm{L}^{\infty}(I)}=O\left(h^{2}\right) .
$$

More precisely we have for $\zeta$ depending on $f$ :

$$
\begin{aligned}
\left(\Pi_{I}(f)-f\right)\left(s_{0}\right) & =\frac{1}{24}\left(f^{\prime \prime}\left(s_{0}\right)-\frac{f^{\prime}\left(s_{0}\right)^{2}}{f\left(s_{0}\right)}\right) h^{2}+O\left(h^{3}\right) \\
\left(\Pi_{I}(f)-f\right)^{\prime}\left(s_{0}\right) & =\zeta h+O(h) \\
\left(\Pi_{I}(f)-f\right)^{\prime \prime}\left(s_{0}\right) & =\left(f^{\prime \prime}\left(s_{0}\right)-\frac{f^{\prime}\left(s_{0}\right)^{2}}{f\left(s_{0}\right)}\right)+O(h) .
\end{aligned}
$$

The key point to prove this theorem is that the parameters of $\Pi_{I}(f)$, namely $a$ and $b$ tend toward a finite limit as the length $h$ of $I$ tends toward 0 . As the parameter $a$ is continuously defined using $m, n$ and $b$ (see for example formula (1.11)), it suffices to prove that the parameter $b$ tends toward a finite limit:

Lemma 3.5. Under all the assumptions and notations of the preceding theorem, we have in a neighbourhood around $h=0$ :

$$
b=-\frac{f^{\prime}\left(s_{0}\right)}{f\left(s_{0}\right)}+O(h)
$$

and the parameter $b$ remains therefore bounded as $h$ tends to 0 . 
Before proving Lemma 3.5, we will introduce some notations: let $m$ and $n$ denote respectively the mass and number density of a function $f$ while $\tilde{m}$ and $\tilde{n}$ will respectively denote the mass and number density of $\Pi_{I}(f)$. Obviously $m, n, \tilde{m}, \tilde{n}, a$ and $b$ depend on the length $h$ of the interval but we will omit to note this dependence. Moreover, we will shorten the notation for the derivatives of $f$ by noting $f_{0}=f\left(s_{0}\right), f_{0}^{\prime}=f^{\prime}\left(s_{0}\right)$ and $f_{0} "=f "\left(s_{0}\right)$.

Proof. Performing a Taylor expansion of $f$ and $\Pi_{I}(f)$ around $s_{0}$ we have the following estimates for the ratios $m / n$ and $\tilde{m} / \tilde{n}$ :

$$
\begin{gathered}
\frac{m}{n}=s_{0}^{3 / 2}\left(1+\frac{1}{8}\left[\frac{1}{2 s_{0}^{2}}+\frac{f_{0}^{\prime}}{s_{0} f_{0}}\right] h^{2}+\mathrm{O}\left(h^{3}\right)\right) \\
\frac{\tilde{m}}{\tilde{n}}=s_{0}^{3 / 2}+\frac{b h}{2\left(\mathrm{e}^{b h / 2}-\mathrm{e}^{-b h / 2}\right)} \int_{-1}^{1}\left[\left(\frac{h u}{2}+s_{0}\right)^{3 / 2}-s_{0}^{3 / 2}\right] \exp (-b h u / 2) \mathrm{d} u .
\end{gathered}
$$

Let us then define:

$$
\begin{gathered}
z(x, h)=\int_{-1}^{1}\left[\left(\frac{h u}{2}+s_{0}\right)^{3 / 2}-s_{0}^{3 / 2}\right] \exp (-x u / 2) \mathrm{d} u \\
K(h)=\frac{m}{n s_{0}^{3 / 2}}-1=\frac{1}{8}\left[\frac{1}{2 s_{0}^{2}}+\frac{f_{0}^{\prime}}{s_{0} f_{0}}\right] h^{2}+\mathrm{O}\left(h^{3}\right) \\
H(x, h)=\frac{x z(x, h)}{h\left(\mathrm{e}^{x / 2}-\mathrm{e}^{-x / 2}\right)}-s_{0}^{3 / 2} \frac{K(h)}{h} .
\end{gathered}
$$

We are now able to give a new characterization for the parameter $b$ :

$$
\frac{\tilde{m}}{\tilde{n}}=\frac{m}{n} \Longleftrightarrow H(b h, h)=0 .
$$

It can be proved that the function $H$ is of class $\mathcal{C}^{2}$ in a neighbourhood around $(0,0)$. Moreover we can compute the following values of $H$ and its derivatives:

$$
\begin{gathered}
H(0,0)=0 \\
\frac{\partial H}{\partial x}(0,0)=-\frac{\sqrt{s_{0}}}{8} \\
\frac{\partial H}{\partial h}(0,0)=-\frac{\sqrt{s_{0}} f_{0}^{\prime}}{8 f_{0}} .
\end{gathered}
$$

The implicit function theorem can then be applied in this case: there exists a neighbourhood $U$ around $(x, h)=(0,0)$ and a function $\varphi$ of class $\mathcal{C}^{2}$ such that:

$$
H(x, h)=0 \Longleftrightarrow x=\varphi(h) \quad \forall(x, h) \in U .
$$

Moreover, we have:

$$
\varphi^{\prime}(0)=-\frac{\partial_{h} H(0,0)}{\partial_{x} H(0,0)}=-\frac{f_{0}^{\prime}}{f_{0}} .
$$

Then we can state that in a neighbourhood around $(0,0)$ we have $x=\varphi(0)+\varphi^{\prime}(0) h+\mathrm{O}\left(h^{2}\right)$, where $\varphi(0)=0$ from (3.11). Furthermore, we noted that $m / n=\tilde{m} / \tilde{n}$ if and only if $H(b h, h)=0$ and we already know that $b$ is uniquely defined. We can then identify $b$ and $\phi(h) / h$ in a neighbourhood around $h=0$ which gives us the desired result.

Lemma 3.6. Under all the assumptions of the theorem (3.4), we have the estimate:

$$
a=f_{0}+O\left(h^{2}\right)
$$


Proof. Using Lemma 3.5, it follows easily from the equality $n=\tilde{n}$ and the Taylor expansions respectively of $n$ and $\tilde{n}$.

The proof of theorem (3.4) yields now no more difficulty since it only relies on identifications of Taylor expansion terms of both $f$ and $\Pi_{I}(f)$ and it is left to the reader. Finally, the exponential approximation we proposed is now proved to be second-order accurate.

Remark 3.7. As it has been stated in the beginning of this paragraph, the results apply to a more general set of functions. If the function $f$ does vanish in $s_{0}$, an extra regularity assumption on $f$ is needed in order to allow the computation of more terms in the Taylor expansion, but no more difficulties appear except technical ones [14].

\subsubsection{Consistency order}

In this part we will focus on the consistency error of the evaporation scheme. As we are studying the error for a discretization step tending to zero, we do not have to take into account the last section (which is infinite). In order to avoid such a problem, we limit our study to compactly supported initial data. Moreover, for the sake of simplicity we will consider functions such that we always have $f_{0} \neq 0$ in Remark 3.7 whereas all the following results may be extended in the general case. We then define the following set of functions:

$$
\mathcal{E}=\left\{f \in \mathbb{C}^{3}\left(\mathbb{R}_{+}\right) / \exists S_{\mathrm{Max}}>0: f(s)>0 \text { for } s<S_{\text {Max }} \text { and } f(s)=0 \text { for } s \geq S_{\text {Max }}\right\}
$$

Obviously, the infinite section will always be chosen as the interval $\left[S_{\text {Max }} ;+\infty[\right.$. We can then give a definition for the consistency error.

Definition 3.8 (Consistency error). Let $f$ be a function in the set $\mathcal{E}$. The consistency error is defined as the difference between the mass and number density computed with the numerical scheme and the exact ones after one time step. As the exact solution of the evaporation equation is known, this difference can be computed as the maximum of the set $\left\{E_{i}\right\}$ defined below:

$$
E_{i}(f)=\frac{1}{\Delta s_{i}}\left\|\operatorname{Err}_{i+1 / 2}(f)-\operatorname{Err}_{i-1 / 2}(f)\right\|_{\infty}+\left|\operatorname{Err} S_{i}(f)\right|
$$

where:

$$
\operatorname{Err}_{i+1 / 2}(f)=\left(\begin{array}{c}
\operatorname{Err}_{i+1 / 2}^{(1)}(f) \\
\operatorname{Err}_{i+1 / 2}^{(2)}(f)
\end{array}\right)=\frac{K}{\Delta t}\left(\begin{array}{c}
\int_{0}^{\Delta t}\left(\left[\Pi_{I_{i}} f\right]\left(s_{i+1}+K t\right)-f\left(s_{i+1}+K t\right)\right) \mathrm{d} t \\
\int_{0}^{\Delta t}\left(s_{i+1}+K t\right)^{3 / 2}\left(\left[\Pi_{I_{i}} f\right]\left(s_{i+1}+K t\right)-f\left(s_{i+1}+K t\right)\right) \mathrm{d} t
\end{array}\right)
$$

and

$$
\operatorname{Err}_{i}(f)=\frac{1}{\Delta s_{i} \Delta t} \int_{0}^{\Delta s_{i}}\left(\left(s_{i}+u\right)^{3 / 2}-\left(s_{i}+u+K \Delta t\right)^{3 / 2}\right)\left(\left[\Pi_{I_{i}} f\right]-f\right)\left(s_{i}+K \Delta t+u\right) \mathrm{d} u
$$

are respectively the errors on the fluxes and on the source term.

Using Theorem 3.4 on the order of approximation with the exponential functions, the following lemma and the theorem on the consistency of the evaporation scheme can be proved.

Lemma 3.9. Let $f$ be a function in the set $\mathcal{E}$. For any integer $i$, let $s_{i+1 / 2}$ denote the middle of the interval $\left[s_{i}, s_{i+1}\right]$ and let $h_{i}$ be the length of the $i$-th section: $h_{i}=\left|s_{i+1}-s_{i}\right|$. Then we have the following estimate:

$$
\operatorname{Err}_{i+1 / 2}^{(1)}(f)=\frac{K}{\Delta t}\left(\frac{\Delta t}{24}\left(f^{\prime \prime}\left(s_{i+3 / 2}\right)-\frac{f^{\prime}\left(s_{i+3 / 2}\right)^{2}}{f\left(s_{i+3 / 2}\right)}\right) h_{i+1}^{2}+O\left(h_{i+1}^{3}\right)\right) .
$$


Proof. The key point of the proof is the estimation of the "projection" error. We know that we have for $s \in] s_{i}, s_{i+1}\left[\right.$ and $h_{i}=s_{i+1}-s_{i}$ :

$$
\begin{aligned}
([\Pi f]-f)(s)= & ([\Pi f]-f)\left(s_{i+1 / 2}\right)+([\Pi f]-f)^{\prime}\left(s_{i+1 / 2}\right)\left(s-s_{i+1 / 2}\right) \\
& +([\Pi f]-f)^{\prime \prime}\left(s_{i+1 / 2}\right) \frac{\left(s-s_{i+1 / 2}\right)^{2}}{2}+\mathrm{O}\left(\left(s-s_{i+1 / 2}\right)^{3}\right) .
\end{aligned}
$$

Reporting this in formula (3.19) for $\operatorname{Err}_{i+1 / 2}^{(1)}(f)$, we have:

$$
\begin{aligned}
\operatorname{Err}_{i+1 / 2}^{(1)}(f)= & \frac{K}{\Delta s \Delta t} \int_{0}^{\Delta t}\left[([\Pi f]-f)\left(s_{i+3 / 2}\right)+([\Pi f]-f)^{\prime}\left(s_{i+3 / 2}\right)\left(s_{i+1}+K t-s_{i+3 / 2}\right)\right. \\
& \left.+([\Pi f]-f)^{\prime \prime}\left(s_{i+3 / 2}\right) \frac{\left(s_{i+1}+K t-s_{i+3 / 2}\right)^{2}}{2}+\mathrm{O}\left(\left(s_{i+1}+K t-s_{i+3 / 2}\right)^{3}\right)\right] \mathrm{d} t .
\end{aligned}
$$

And recalling the estimates from Theorem 3.4 gives the desired result.

Theorem 3.10. Let $f \in \mathcal{E}$. We suppose our discretization in size is done the following way: $\left(s_{i}\right)_{1 \leq i \leq N}$ with $s_{N}=S_{\max }$. Then the scheme (2.17) is consistent of order 1 with the equation, and is consistent of order 2 if the discretization step is constant.

Proof. The key point is the computation of the difference $\operatorname{Err}_{i+1 / 2}^{(1)}(f)-\operatorname{Err}_{i-1 / 2}^{(1)}(f)$. We will use the notation $\tau(s)=f^{\prime \prime}(s)-\frac{f^{\prime}(s)^{2}}{f(s)}$ and $h=\max _{i}\left(h_{i}\right)$. From lemma (3.9), we get:

$$
\operatorname{Err}_{i+1 / 2}^{(1)}(f)-\operatorname{Err}_{i-1 / 2}^{(1)}(f)=\frac{K}{24}\left(\tau\left(s_{i+3 / 2}\right) h_{i+1}^{2}-\tau\left(s_{i+1 / 2}\right) h_{i}^{2}+\mathrm{O}\left(h^{3}\right)\right) .
$$

Furthermore, if the discretization step is constant, there exists $\xi \in] s_{i+1 / 2}, s_{i+3 / 2}[$ such that:

$$
\operatorname{Err}_{i+1 / 2}^{(1)}(f)-\operatorname{Err}_{i-1 / 2}^{(1)}(f)=\frac{K}{24}\left(\tau^{\prime}(\xi)\left(s_{i+3 / 2}-s_{i+1 / 2}\right) h^{2}+\mathrm{O}\left(h^{3}\right)\right)=\mathrm{O}\left(h^{3}\right) .
$$

We have the same type of inequality for $\operatorname{Err}_{i+1 / 2}^{(2)}(f)-\operatorname{Err}_{i-1 / 2}^{(2)}(f)$. It remains to prove an order estimate on $\operatorname{Err} S_{i}$ : this is always of order $h^{2}$. Indeed, this comes straight from Theorem 3.4 and the fact that:

$$
\left|\left(s_{i}+u\right)^{3 / 2}-\left(s_{i}+u+K \Delta t\right)^{3 / 2}\right|=\mathrm{O}(K \Delta t)
$$

and $K \Delta t \leq \min \left(h_{i}\right)$. Finally, integrating these results in formula (3.18) concludes the proof.

\subsubsection{Comments on the evaporation scheme}

Here are discussed some few remarks on the evaporation scheme. First, scheme (2.17) is non-linear since the "projection" $\Pi_{I}$ is highly non-linear. Moreover, this scheme is neither monotonic nor total variation diminishing. This comes from the fact that the parameter $b$ can take any real value so that the exponential family is able to represent any positive density function.

The scheme has been derived so that both number and mass density are always naturally positive but not $L^{\infty}$ bounded. However, if we assume that the parameter $b$ remains bounded we can ensure a $L^{\infty}$ bound on our scheme. Practically, this assumption is not restrictive since in all the computations performed so far, the numerical parameter $b$ remained bounded, although this property has not been theoretically proven yet.

Proposition 3.11. Let $T>0$ and assume that the parameter $b$ remains bounded by a constant $B$ for all time and size steps. Then, if the initial condition (number and mass density) is $L^{\infty}$-bounded, the number and mass 
density remains bounded for all time $t \in[0, T]$, given the fact that the time step satisfies the CFL condition $K \Delta t \leq \Delta s$.

Proof. Let $\alpha$ be the inverse of the CFL constant. Then, the results comes from the fact that the derivative with respect to the first variable of the function $g$ defined in (2.25) is bounded by a constant $M_{\alpha}$ (see Rem. 2.6). Indeed, recalling the notations from the numerical scheme (2.23) we have:

$$
n_{i}^{p+1}=n_{i}^{p}\left(1-g\left(b_{i} K \Delta t, \alpha\right)\right)+n_{i+1}^{p} g\left(b_{i} K \Delta t, \alpha\right)+n_{i+1}^{p}\left(g\left(b_{i+1} K \Delta t, \alpha\right)-g\left(b_{i} K \Delta t, \alpha\right)\right)
$$

yielding the following inequality, with $N^{p}=\max _{i}\left(\left|n_{i}^{p}\right|\right)$,

$$
N^{p+1} \leq N^{p}\left(1+g\left(b_{i+1} K \Delta t, \alpha\right)-g\left(b_{i} K \Delta t, \alpha\right)\right) \leq N^{p}\left(1+2 B M_{\alpha} K \Delta t\right) .
$$

Using a discrete Gronwall lemma, the bound on the number density is straightforward. To conclude the proof, we only have to remark that the mass density is then also bounded since the ratio $m / n$ is proved to be bounded in any section (see Prop. 3.3).

Remark 3.12. If the parameter $b$ is assumed to remain bounded by a constant $B$, the total variation (TV) of the numerical solution also remains bounded by a constant depending on $B$ during the approximation step. Nevertheless, this is not enough in order to prove that the scheme is a TV bounded one and then to prove the convergence, although these last two points seem to be numerically verified. A similar kind of problem appears when using a "essentially non oscillatory" (ENO) scheme $[19,28]$, for which the ENO reconstruction step is total variation bounded but no global TV bound is provided, although numerical results seem to suggest that such a bound exists [28].

\section{Numerical ReSults}

We here present some numerical results using the "revisited multi-fluid method" for the evaporation phenomena. The aim is to compare the accuracy of the hybrid method with the ones of two other schemes. In order to evaluate the accuracy of a numerical scheme, two quantities will be checked, the first being the Sauter mean radius which is defined as:

$$
r_{s m d}=\frac{1}{3 \sqrt{4 \pi}} \frac{\int_{0}^{+\infty} s^{3 / 2} f(s) \mathrm{d} s}{\int_{0}^{+\infty} s f(s) \mathrm{d} s} .
$$

The second quantity tested is the mass of non-evaporated liquid. These two quantities are very important considering the application of sprays in the combustion domain. Both quantities will be checked either as a function of time (0D case) or as a function of position (1D stationary case). In the last case, evaporation is coupled with the transport phenomena.

\subsection{0-dimensional test-case}

Let us begin with a test-case which will enable us to point out the ability of our evaporation scheme to simulate accurately the evaporation phenomena, governed by the kinetic equation:

$$
\left\{\begin{aligned}
\partial_{t} f+K \partial_{s} f & =0 \\
f(0, s) & =f_{0}(s) .
\end{aligned}\right.
$$

Three schemes will be compared to the exact solution which can be computed exactly: the classical multi-fluid method [24,37], a MUSCL method [38] performed on the number density of the droplets and the improved multi-fluid method using two moments, this last one being called the "exponential method" in all the following. More precisely, the MUSCL method is performed using a discretization of the following equation on the number density:

$$
\partial_{t} n-K \partial_{s} n=0
$$



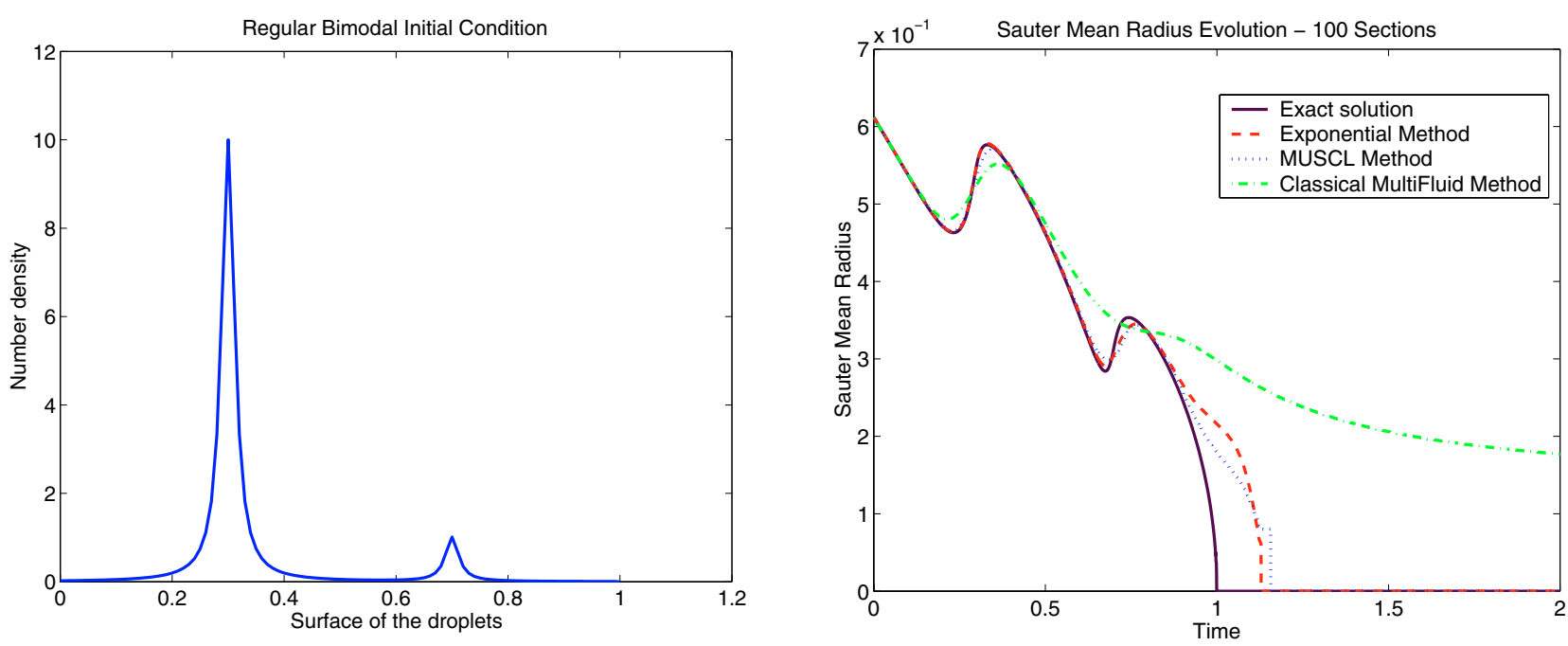

FIGURE 2. Initial condition and Sauter mean radius evolution using 100 sections.

Remark 4.1. The MUSCL method could also have been applied on the conservation equation for the number density but such a method leads to numerical difficulties since this equation is singular when the surface of the droplets tends to zero. Therefore, rebuilding the number density from the mass density using such an approach yields too many numerical errors.

Two kinds of initial data are chosen for this test-case. The first one (Fig. 2, left) is regular and part of the space $\mathcal{E}$ with $S_{N}=1$ (see Eq. (3.17)). Moreover, this initial data is taken with quite a complicated shape, corresponding to a bimodal droplet size distribution. Choosing such initial data yields a non-trivial evolution of the Sauter mean radius and points out the ability of the different schemes to take into account the "subsectional" variations of the number density. The second initial data (Fig. 7, left) is a step function which will ensure that the schemes used can deal with discontinuities.

Remark 4.2. For the sake of clarity, we choose to normalize our results: all the surfaces of the droplets will be taken between 0 and 1 and the time it takes for the whole distribution to vanish completely is assumed to be 1 .

\subsubsection{Regular initial data}

We first consider the regular initial data which may be seen as a bimodal test-case with a representation of two sizes. The evolution of the Sauter mean radius can be decomposed in three stages. It first decreases until the first group of droplets disappears, giving more importance to the second group of droplets and then yielding a quick increase of the mean radius. The Sauter mean radius then decreases again until the second group of droplets also disappears, letting the Sauter mean radius go to zero after a slight last increase (Fig. 2, left).

The first figures represent the evolution of the Sauter mean radius for all methods using 100 sections (Fig. 2, right), 10 and 5 sections (Fig. 3). The 100 sections case shows the convergence of all methods (while the classical multi-fluid method would need even more sections) and the other two cases (10 and 5 sections) show the ability of these methods to predict at least the global behaviour of the evolution with a few number of sections. One can see the great improvement obtained by using second-order methods.

Only the multi-fluid-type methods take into account the conservation equation on the mass, the MUSCL method does only predict its value using the rebuild affine number density. One can see on the left of Figure 4 that the approximation is quite good for all methods with a great number of section but that the error for the MUSCL method grows quickly as the number of section decreases. Indeed, for a few number of sections (Fig. 5, left), the first order method and the MUSCL method have the same order accuracy on mass and for an even coarser mesh (Fig. 6, left), the error on the rebuild mass for the MUSCL method is much bigger than 

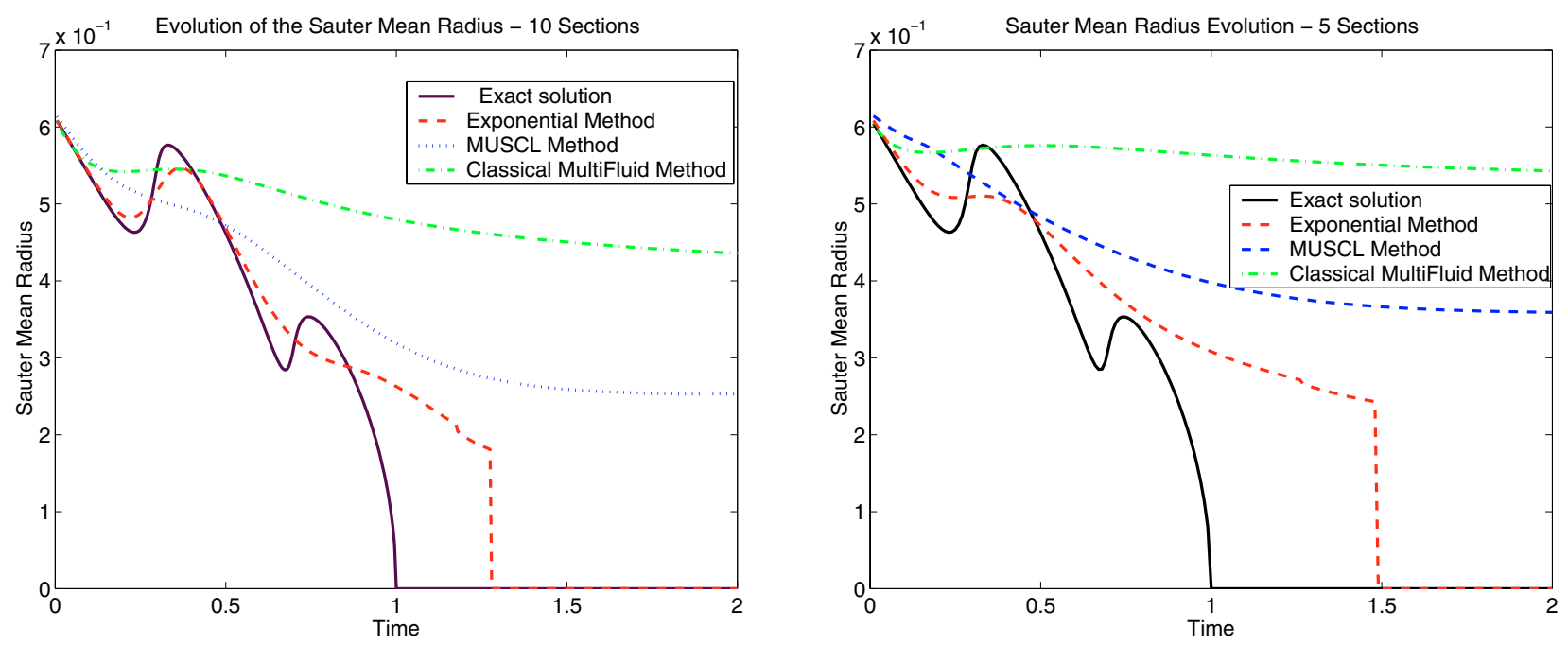

FIGURE 3. Sauter mean radius evolution -10 and 5 sections.
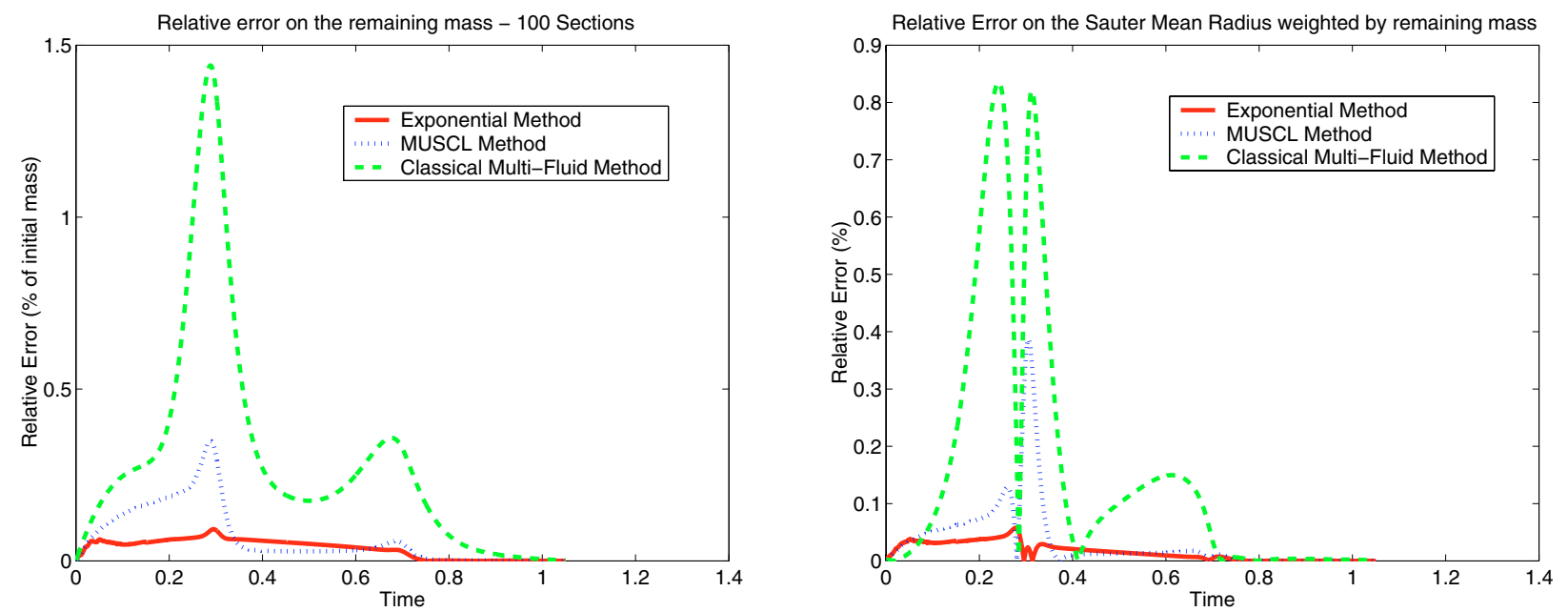

FiguRE 4. Relative errors on remaining mass (left) and Sauter mean radius (right) - 100 sections.

these for the classical multi-fluid and exponential methods. One can remark that the accuracy of the improved multi-fluid method remains quite good even on a coarse grid. It is shown to be a real improvement of the classical multi-fluid since it does always provide a better approximation for the mass density evolution.

On the right part of Figures 4-6, the evolution of the Sauter mean radius weighted by the remaining mass (according to the method considered) is represented. Such a quantity is relevant to compute since for all methods, the error on the Sauter mean radius becomes important when most of the droplets have disappeared, due to the intrinsic numerical errors. Since the effects of the liquid phase on its neighbourhood is proportional to its local density, Figures 4-6 show the error on the Sauter mean radius when it does have an important meaning. One can see that the improvement on the number density distribution due to the second order accuracy for the MUSCL method is completely masked by the poor estimate on the remaining mass on a coarse mesh since both the classical multi-fluid method and the MUSCL method do have the same order of error estimates (Figs. 5, 6). 

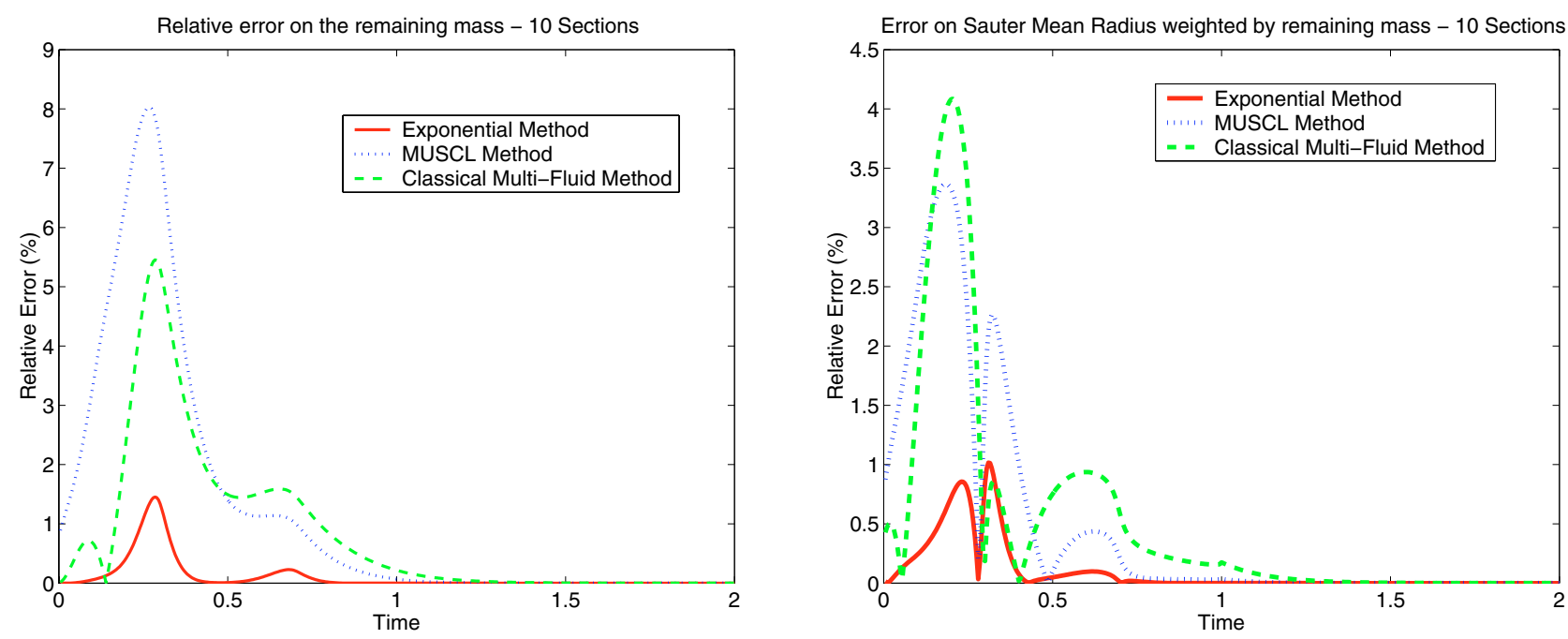

Figure 5. Relative errors on remaining mass (left) and Sauter mean radius (right) - 10 sections.
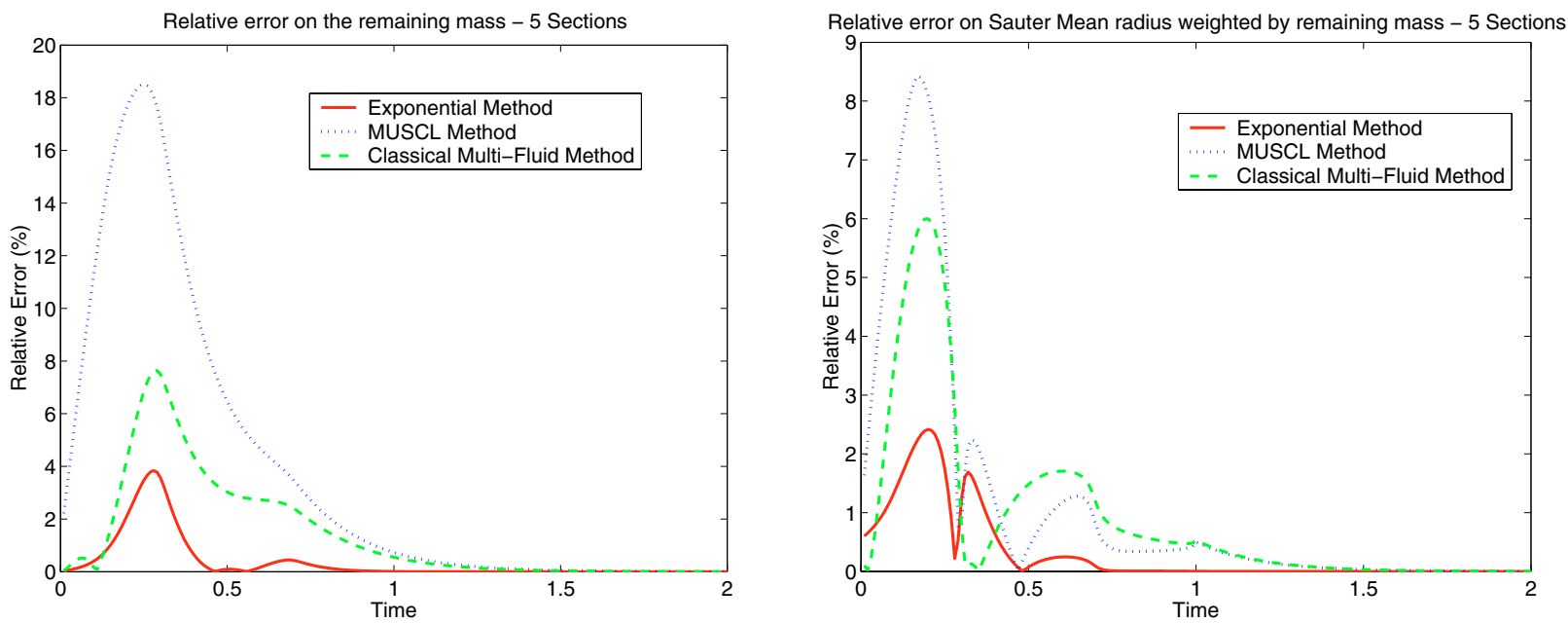

FiguRE 6. Relative errors on remaining mass (left) and Sauter mean radius (right) - 5 sections.

These results emphasize the importance of preserving two moments since the exponential method does have a good error estimate on both quantities even for a coarse mesh.

\subsubsection{Step function}

We now take a step function as initial condition. As all the results proved in part 3 do concern regular functions, here we are testing the ability of the improved multi-fluid method to deal with discontinuous functions. The results on the Sauter mean radius evolution are quite the same as for the regular bimodal case since the exponential method shows an improved accuracy on all fine (100 sections), medium (10 sections) and coarse (5 sections) meshes. In particular, focusing on the time it takes for the whole distribution to completely evaporate, it has to be noted that the exponential method provides a relevant estimate for all meshes whereas the MUSCL method leads to a completely different estimate between the fine (Fig. 7) and medium or coarse (Fig. 8) meshes. 

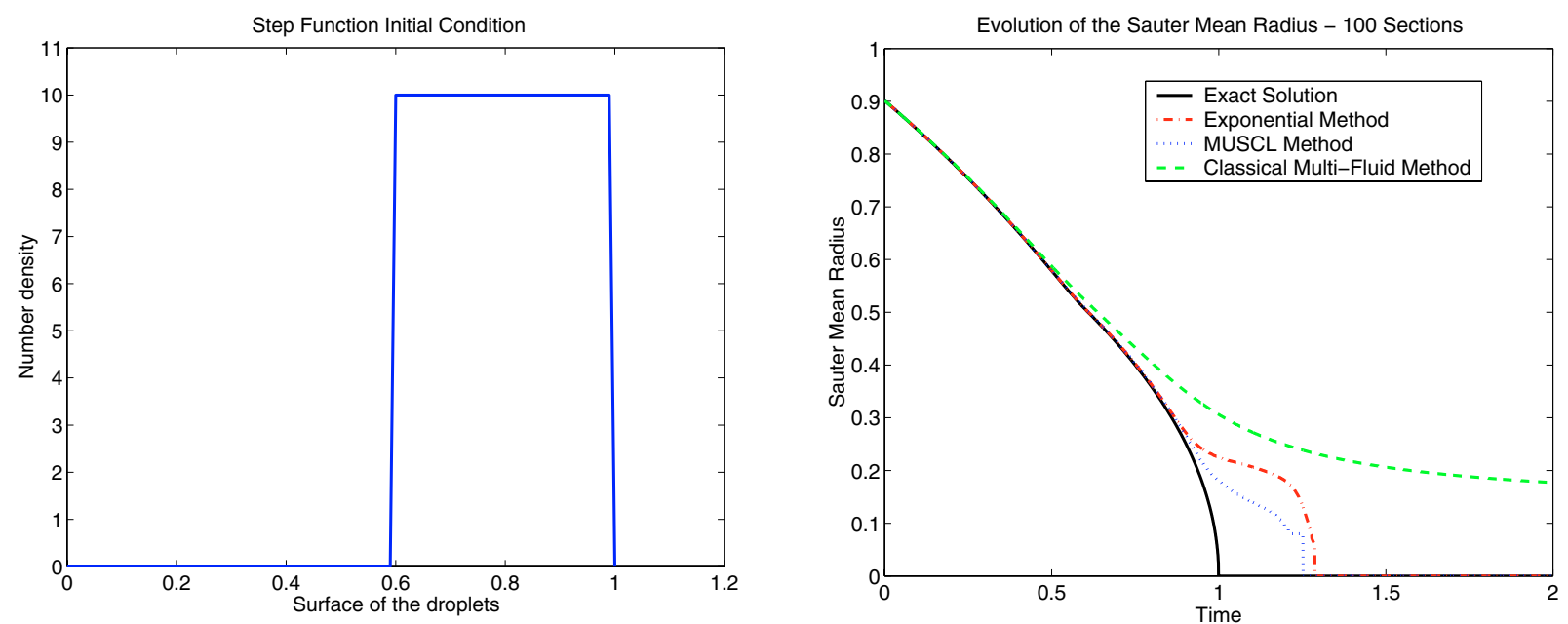

FIGURE 7. Initial condition and evolution of the Sauter mean radius for 100 sections.
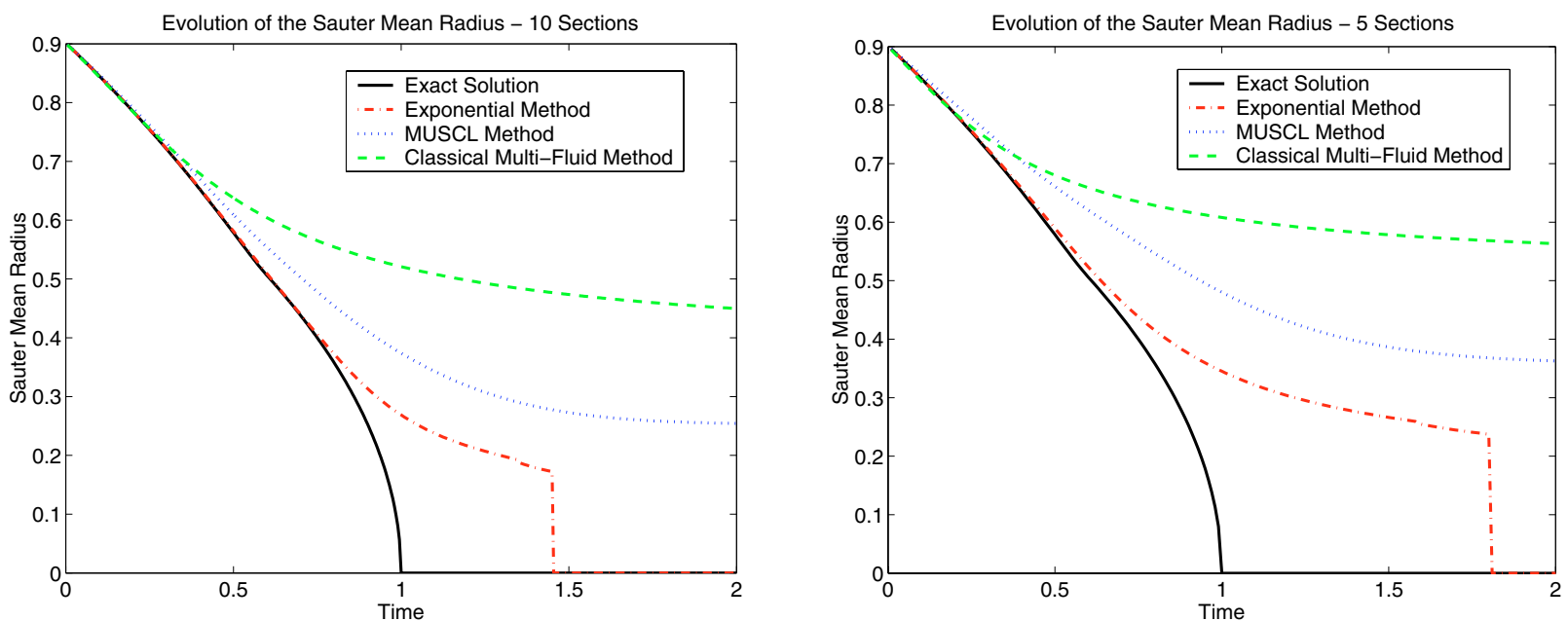

Figure 8. Evolution of the Sauter mean radius -10 and 5 sections.

As in the preceding bimodal test-case when using medium or coarse meshes (Figs. 10 and 11), the secondorder MUSCL method does not show any real improvement of accuracy on the mass density, whereas the exponential method does provide a strongly reduced error in figures (Figs. 10 and 11). This ability of the exponential method to deal with discontinuities when a few number of sections is used may come from its non-TVD property.

The results on the Sauter mean radius evolution can be commented in the same manner as in the previous case, emphasizing once more the improvement due to the preservation of two moments in any section. Indeed, it is worth noting that the difference on the mass estimate for both classical and improved multi-fluid methods is even more important in Figure 11 (left) than in Figure 6 (left), yielding an important improvement of the accuracy on the Sauter mean radius evolution (Fig. 11, right). 

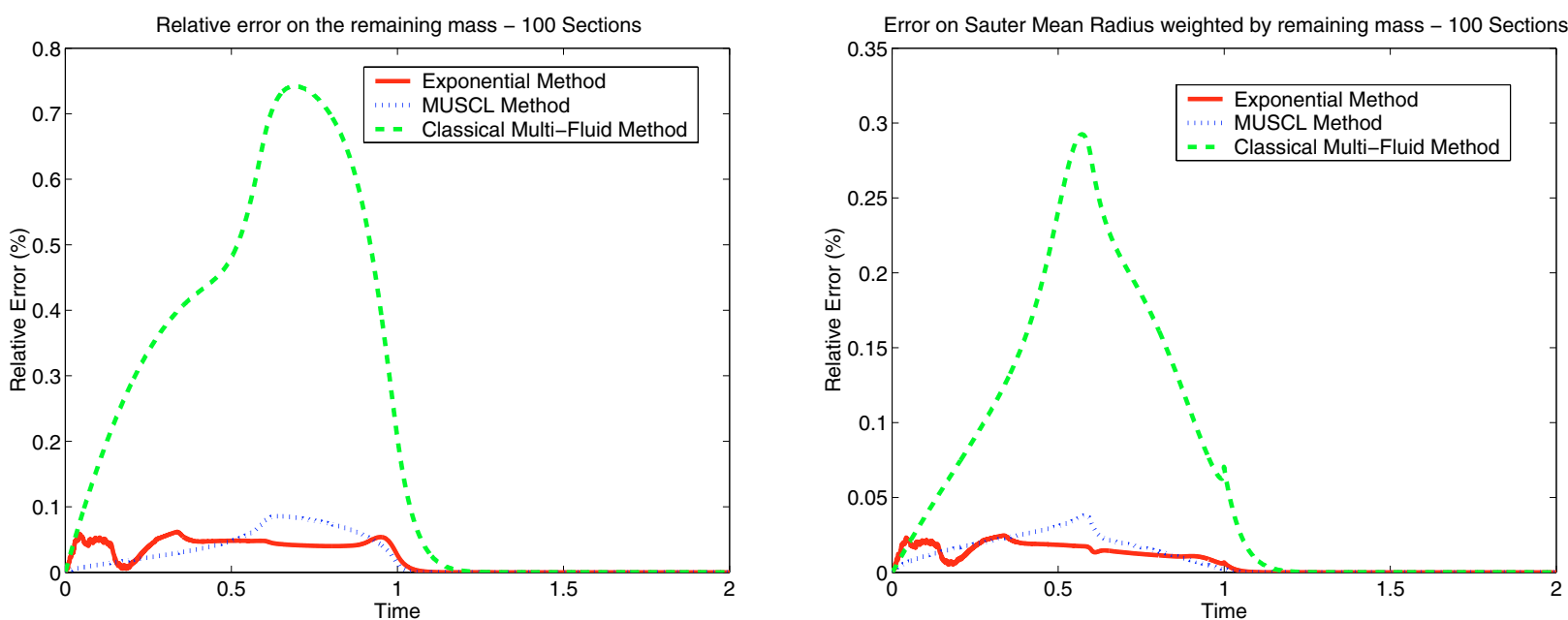

Figure 9. Relative errors on remaining mass (left) and Sauter mean radius (right) - 100 sections.
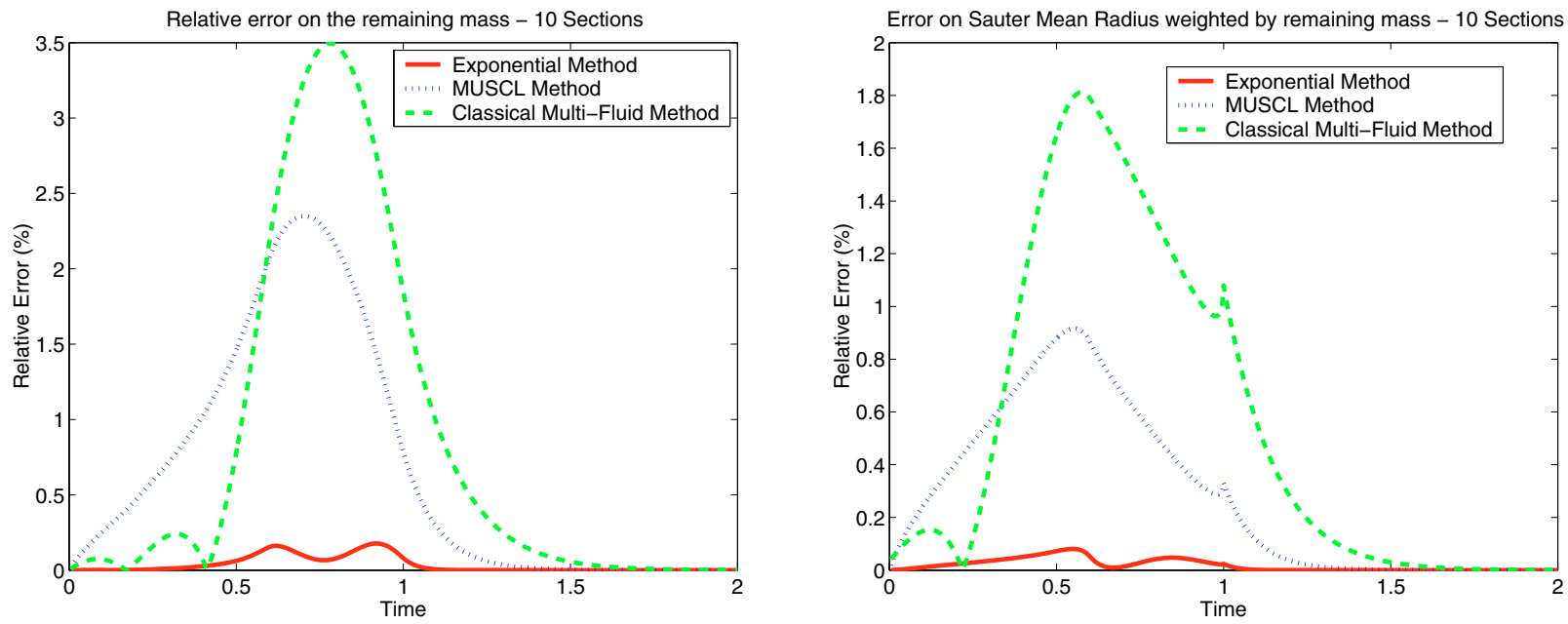

FiguRE 10. Relative errors on remaining mass (left) and Sauter mean radius (right) - 10 sections.

\subsection{1-dimensional test-case}

This test-case takes into account the evaporation phenomenon, the drag force and the transport of the droplets. The transport scheme used is the one described in part (2.2). We aim at performing comparisons on the steady solution of the kinetic equation which is the solution of the following equation as a function of position, velocity and surface of the droplets:

$$
\left\{\begin{aligned}
v . \partial_{x} f+\partial_{v}\left(\frac{U_{g}-v}{\gamma s} f\right)-K \partial_{s} f & =0 \\
f(x=0, s, v) & =f_{0}(s, v) .
\end{aligned}\right.
$$

We will only consider the case of a regular monomodal initial condition (see Fig. 12). 

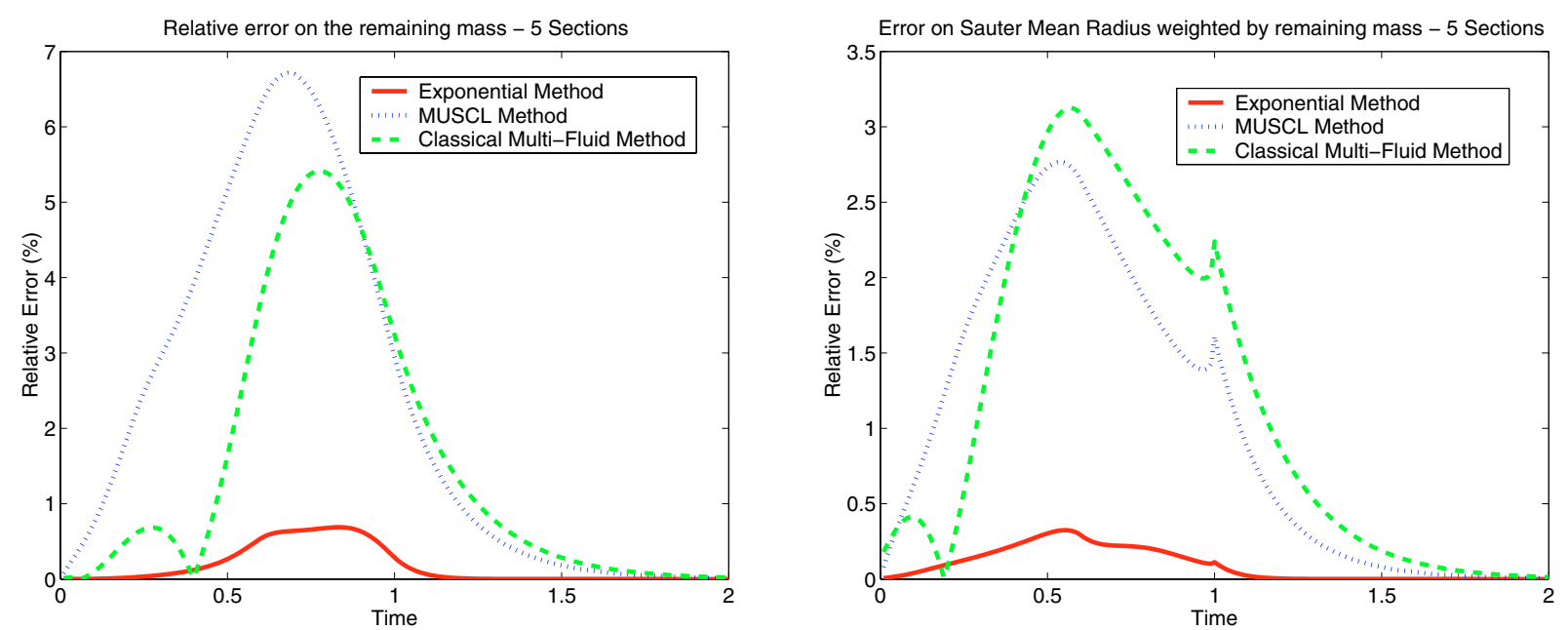

FIGURE 11. Relative errors on remaining mass (left) and Sauter mean radius (right) - 5 sections.
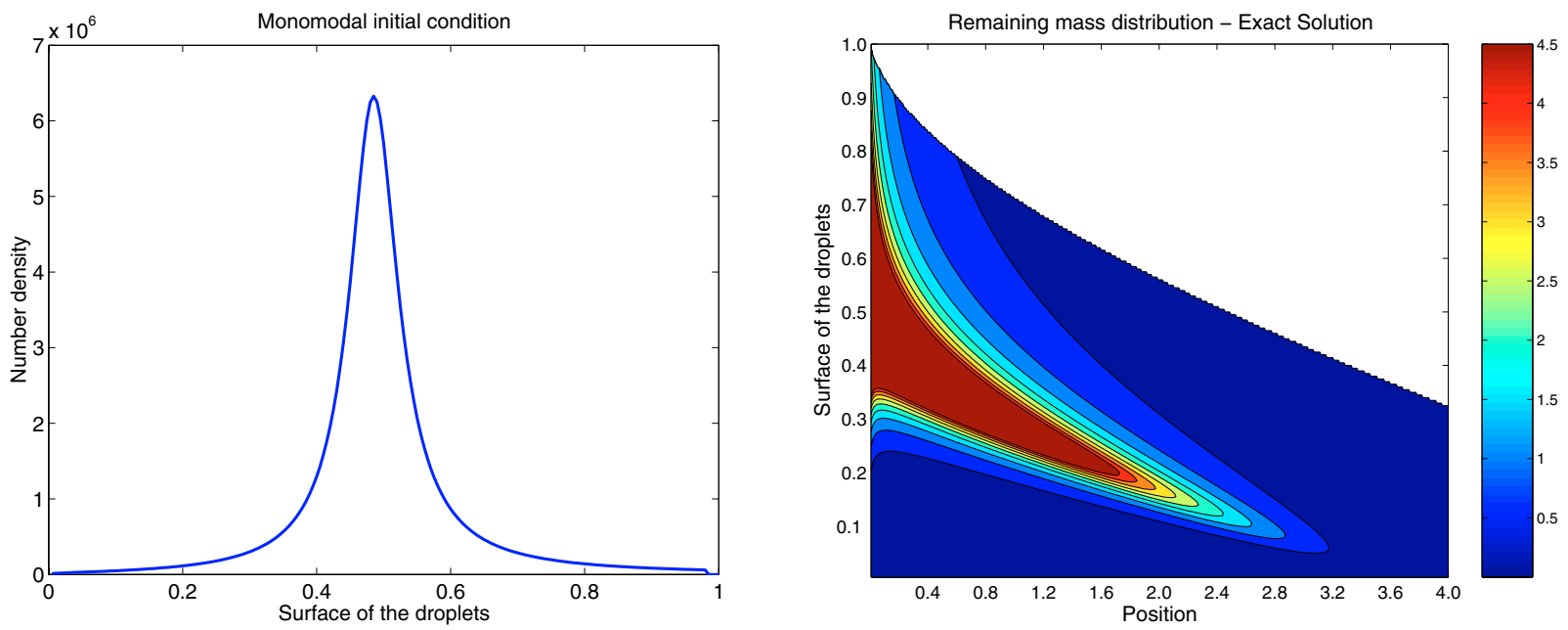

FIGURE 12. Regular initial condition and mass density repartition for the exact solution.

\subsubsection{Reference solution}

Since there exists no analytic solution to the preceding equation we have to compute this one numerically. In Lagrangian coordinates, the evolution of the surface and velocity of droplets writes:

$$
\frac{\mathrm{d} s}{\mathrm{~d} x}=-\frac{K}{v}, \quad \frac{\mathrm{d} v}{\mathrm{~d} x}=\frac{U_{g}-v}{\gamma v s}, \quad \frac{\mathrm{d} v}{\mathrm{~d} s}=\frac{U_{g}-v}{-K \gamma s}
$$


Using the last equation we can deduce the value of the velocity of one droplet knowing its actual size and initial size and velocity:

$$
\begin{aligned}
& \ln \left(\frac{U_{g}-v}{U_{g}-v_{0}}\right)=\frac{1}{\gamma K} \ln \left(\frac{s}{s_{0}}\right) \\
& v=U_{g}-\left(U_{g}-v_{0}\right)\left(\frac{s}{s_{0}}\right)^{\frac{1}{\gamma K}} .
\end{aligned}
$$

Let $\beta=1 /(\gamma K)$. Using the first equation on the evolution of the surface, we easily show that the surface of the droplet at the position $x$ knowing its initial surface and density is solution of:

$$
s(x)^{\beta+1}-(\beta+1) \frac{U_{g}}{U_{g}-v_{0}} s_{0}^{\beta} s(x)+\frac{\beta U_{g}+v_{0}}{U_{g}-v_{0}} s_{0}^{\beta+1}=(\beta+1) \frac{s_{0}^{\beta} K x}{U_{g}-v_{0}} .
$$

One may check that for a given triplet $(x, s(x), v(x))$ there exists one and only one couple $\left(s_{0}, v_{0}\right)$ satisfying this equation. In order to simplify this inversion problem a little, we will assume that all droplets at $x=0$ have the same velocity $v_{0}>0$. It is then quite obvious that for any $x>0$ and $s>0$ the corresponding velocity lies within the interval $\left[v_{0}, U_{g}\right]$.

Let us consider $F_{x}$ the function that gives $(s, v)$ obtained at position $x$ starting from a droplet of characteristics $\left(s_{0}, v_{0}\right)$. We have:

$$
I=\int_{s_{1}}^{s_{2}} \int_{v_{1}}^{v_{2}} f(x, s, v) \mathrm{d} s \mathrm{~d} v=\int_{s_{1}}^{s_{2}} \int_{v_{1}}^{v_{2}} f\left(F_{x}\left(s_{0}, v_{0}\right)\right) \mathrm{d} s \mathrm{~d} v .
$$

Performing the change of variable $(s, v)=F\left(s_{0}, v_{0}\right)$ we have for $D=F^{-1}\left(\left[s_{1}, s_{2}\right] \times\left[v_{1}, v_{2}\right]\right)$ :

$$
I=\iint_{D} f_{0}(t, w)\left|\begin{array}{ll}
\frac{\partial F_{x, 1}}{\partial_{s}} & \frac{\partial F_{x, 1}}{\partial_{v}} \\
\frac{\partial F_{x, 2}}{\partial_{s}} & \frac{\partial F_{x, 2}}{\partial_{v}}
\end{array}\right| \mathrm{d} t \mathrm{~d} w .
$$

Denoting by $J(x)$ the Jacobian, we have the following equation:

$$
J^{\prime}(x)=J(x)\left(\frac{\partial^{2} F_{x, 1}}{\partial s \partial x}+\frac{\partial^{2} F_{x, 2}}{\partial v \partial x}\right)
$$

That gives, knowing that $J(0)=1$,

$$
J(x)=\exp \left(-\int_{0}^{x} \frac{U_{g} \mathrm{~d} x}{\gamma s(x) v(x)^{2}}\right) .
$$

Recalling the relation between $x$ and $s$ knowing $s_{0}$ and $v_{0}$ we can perform the change of variable $u=s(x)$, using the fact that:

$$
\begin{gathered}
x^{\prime}(s)=\frac{U_{g}-v_{0}}{K}\left(\frac{s}{s_{0}}\right)^{\beta}-\frac{U_{g}}{K} \\
J(s)=\exp \left(-\int_{s}^{s_{0}} \frac{\beta U_{g}}{u\left|U_{g}+\left(v_{0}-U_{g}\right)\left(\frac{u}{s_{0}}\right)^{\beta}\right|} \mathrm{d} u\right) .
\end{gathered}
$$

We are now able to compute numerically the number density at $x=0$ using both numerical inversion and numerical integration: $f(x, s, v)=f\left(0, s_{0}\right) J\left(s, v_{0}\right)$. We will consider that the number density obtained using a fine discretization of the space $(x, s)$ is our reference solution. 

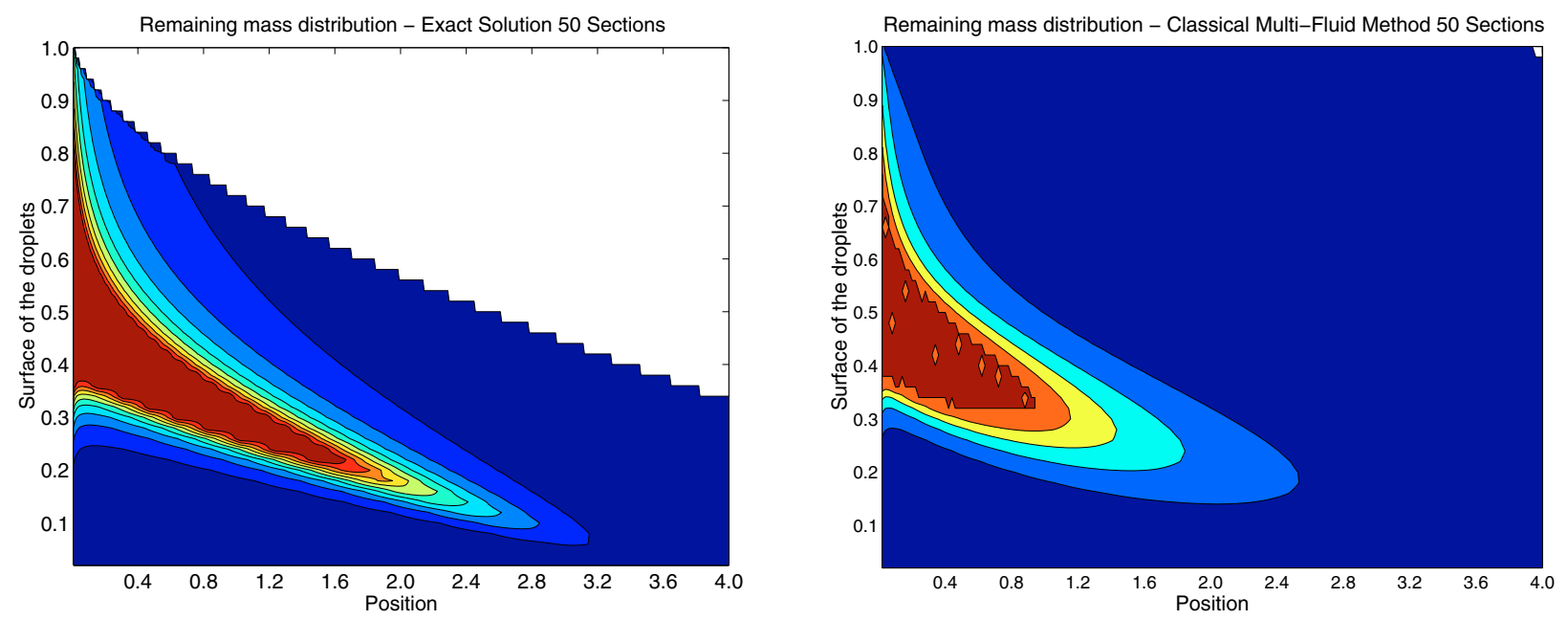

Figure 13. Distribution of the mass density - exact solution and classical multi-fluid method using 50 sections.
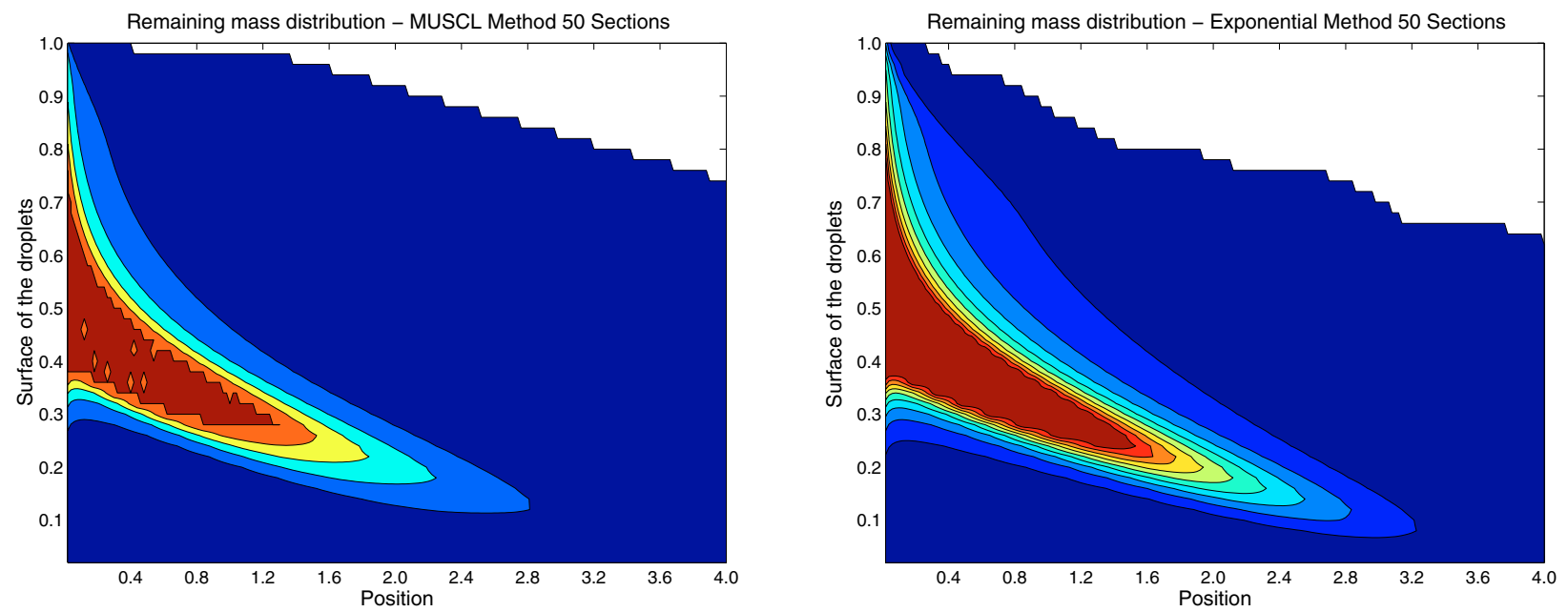

FIGURE 14. Distribution of the mass density - MUSCL method and exponential method using both 50 sections.

\subsubsection{Numerical computations using a regular initial condition}

In this section, a simulation using a monomodal regular initial condition is performed, the initial condition given in the left part of Figure 12. The study of the exact solution (whose mass density distribution is drawn in the right part of Fig. 12) emphasizes the coupling between the drag force and the evaporation phenomenon since the droplet velocity is obviously growing as the surface of the droplets decrease until it reaches the velocity of the surrounding gas.

Here, we compare the same three numerical methods as in the 0D-case and to this end, we first check the distribution obtained for the mass density in order to see whether or not the schemes are able to give the global behaviour of the spray. In Figures 13 and 14 the mass density is presented as a function of position and size of the droplets. Obviously, the same colour table as for Figure 12 has been applied to all drawings so that the comparisons are relevant. In order to be able to compare the effects of the schemes only, the exact solution has 

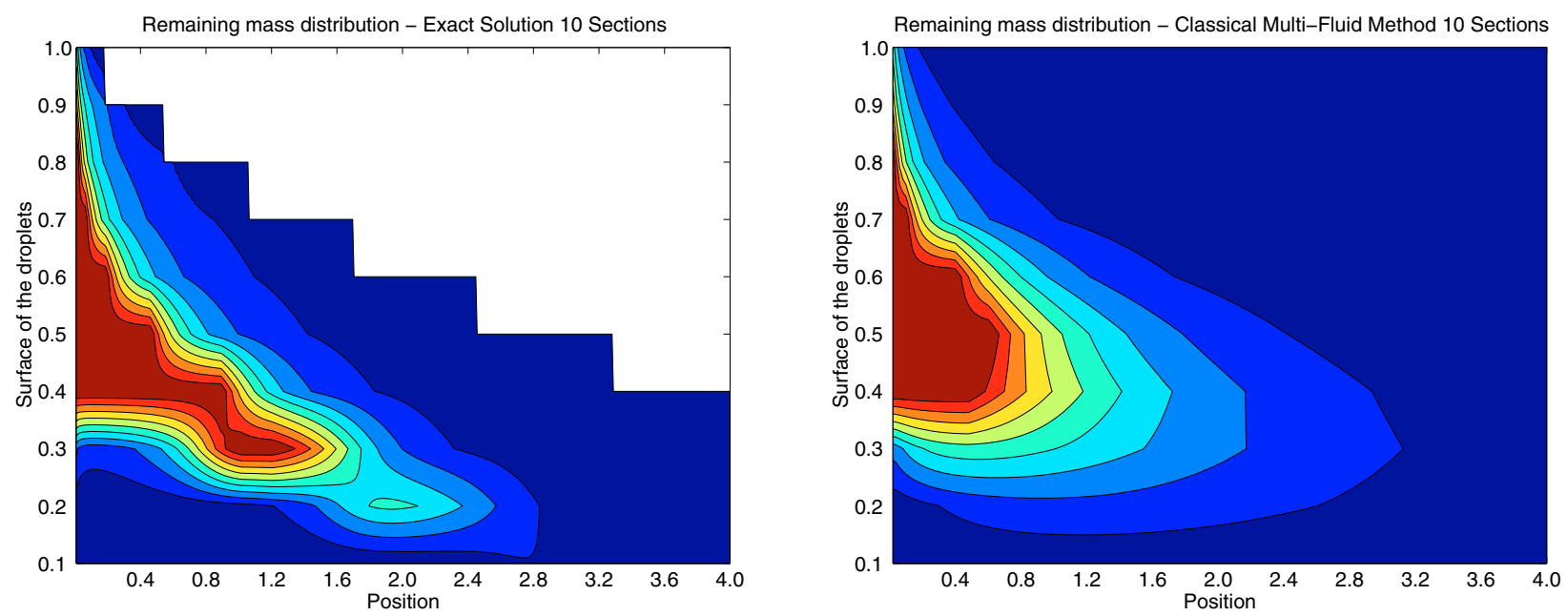

FIGURE 15. Distribution of the mass density - exact solution and classical multi-fluid method using 10 sections.
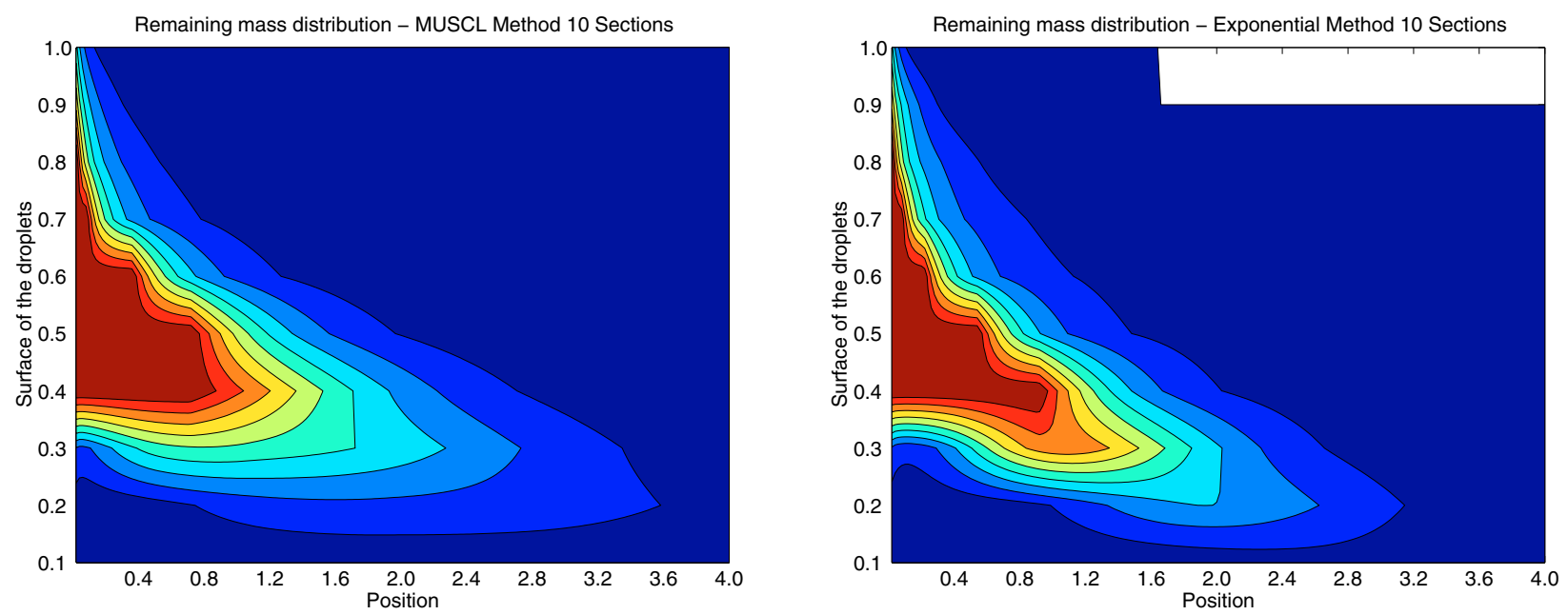

FiguRE 16. Distribution of the mass density - MUSCL method and exponential method using both 10 sections.

been discretized using the same number of sections. Please note that the white colour denotes the absence of any droplet so that we have an idea of the ability of the scheme to limit the artificial diffusion. One can check the fact that the exponential method gives a sharper approximation of the mass density field than the other two while the MUSCL method also shows a real improvement compared to the classical multi-fluid method. In particular, checking the vanishing of the second density level we can see that it occurs around the position 3.2 for the exact solution as well as for the exponential simulation but this density level does vanish earlier for the two other simulations.

We now turn to the same comparisons but with only 10 sections. As the exact solution has been discretized with only 10 sections, the distribution of the mass density looks rougher but the global shape is the same. One more time, the exponential method does provide a relatively good estimate of the mass density field, especially concerning the localisation of high mass density. 

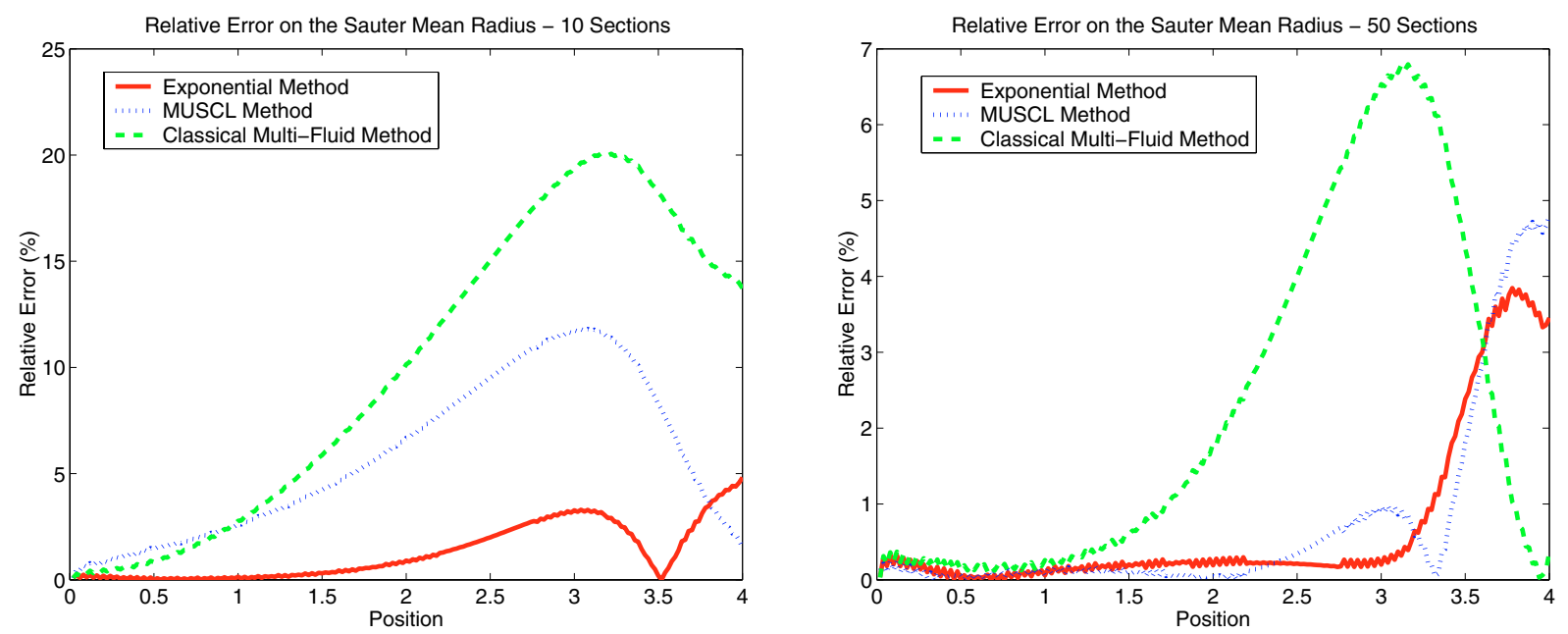

Figure 17. Relative error on the Sauter mean radius - 10 and 50 sections.
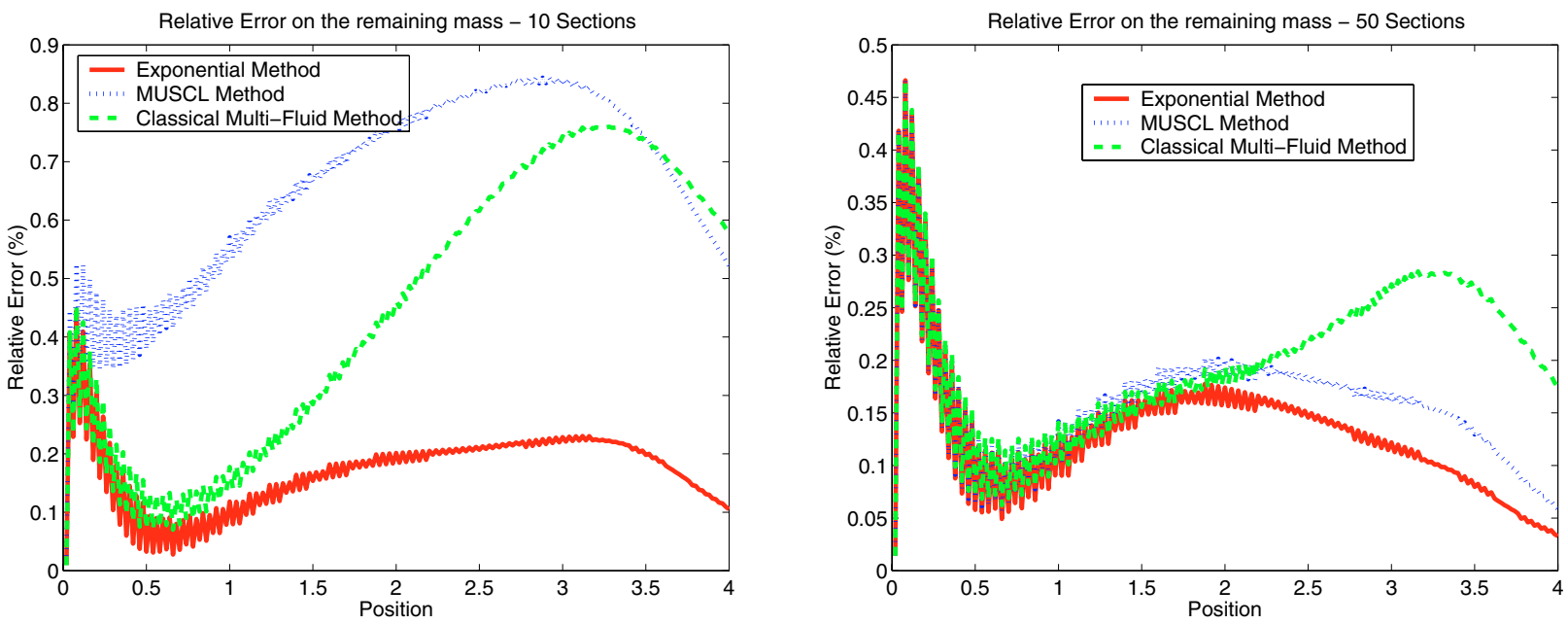

FiguRE 18. Relative error on the remaining mass -10 and 50 sections.

In Figures 17-19, the same conclusions on the numerical simulation hold since the exponential numerical method enables to reduce the number of sections while keeping a rather good accuracy on both the remaining mass and the Sauter mean radius. The error of the first velocity moment $\bar{v}$ is also presented, where $\bar{v}$ is defined by

$$
\bar{v}(x)=\frac{\iint_{s, v} v f(x, s, v) \mathrm{d} s}{\iint_{s, v} f(x, s, v) \mathrm{d} s} .
$$

It can be observed that the global behaviour of the error for the MUSCL method as well as for the classical multi-fluid method changes as the number of section decreases, showing an important dependence on the number of sections. On the contrary, the error for the exponential method is just slightly increased. This effect can be seen as a consequence of the ability of the improved multi-fluid method to always provide the general way of evolution of the distribution even for a few number of section whereas for the two other methods, the rebuilt 

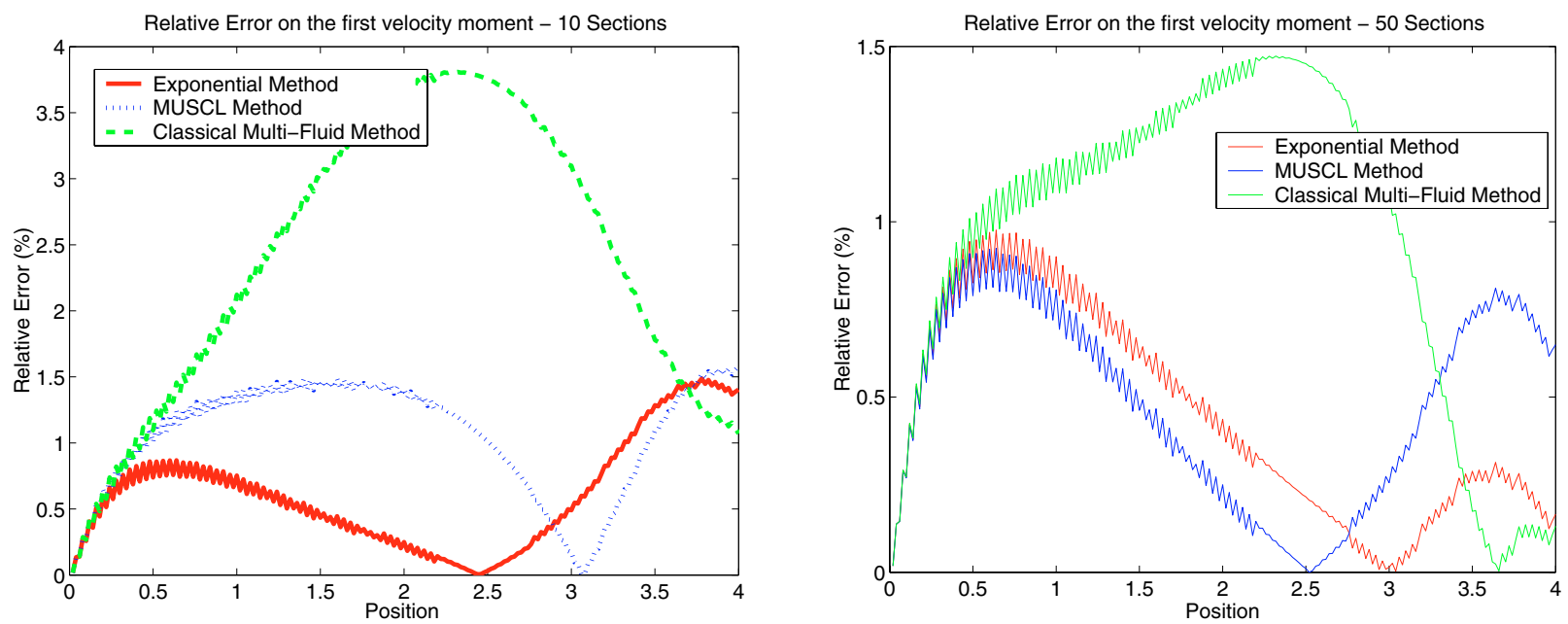

FIGURE 19. Relative error on the first velocity moment (see (4.9)) - 10 and 50 sections.
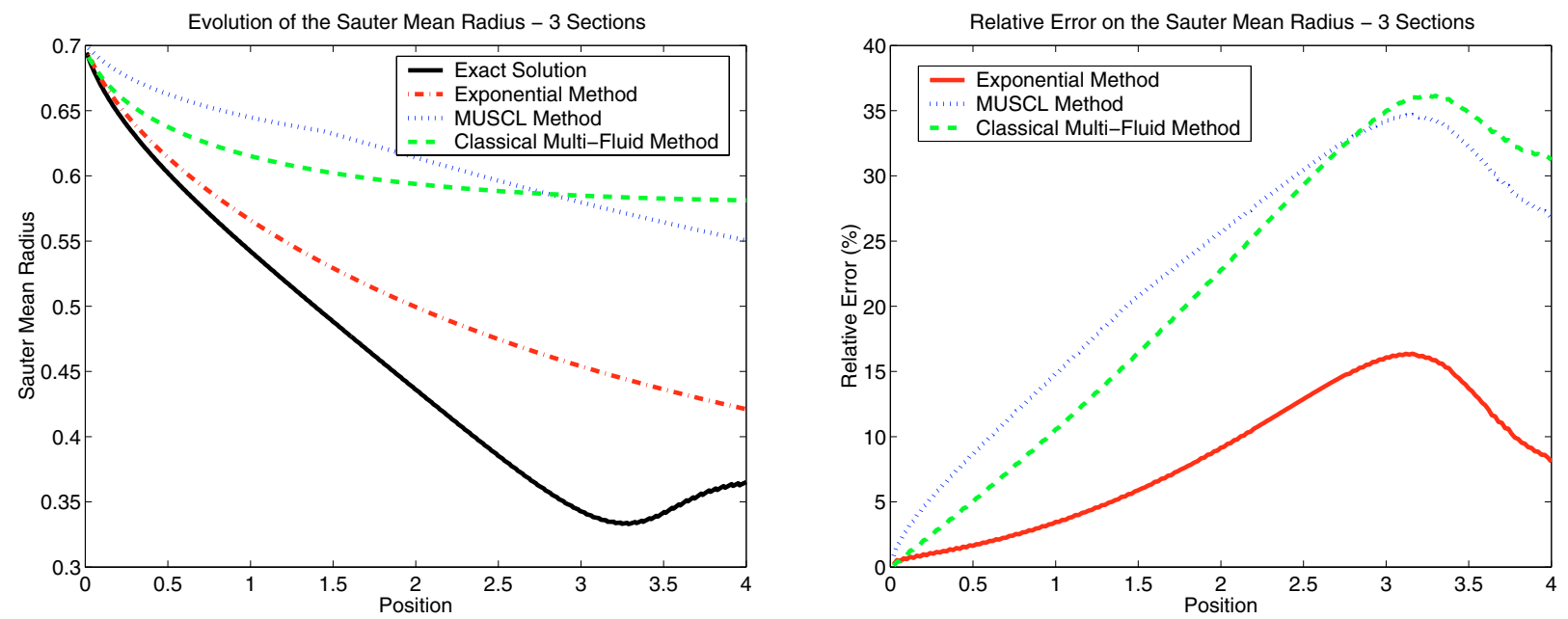

FiguRE 20. Relative errors on mass, first velocity moment and Sauter mean radius for 3 sections.

mass density using the MUSCL method or the rebuilt number density using the classical multi-fluid method introduce numerical errors, inducing different behaviours for the Sauter mean radius.

In order to show that the exponential method is able to deal with very few sections, a numerical simulation has been performed using only 3 sections (Figs. 20 and 21). At this level of discretization, the regularity of the initial condition is no more a relevant aspect and the aim of such a numerical test is to describe a limiting case 

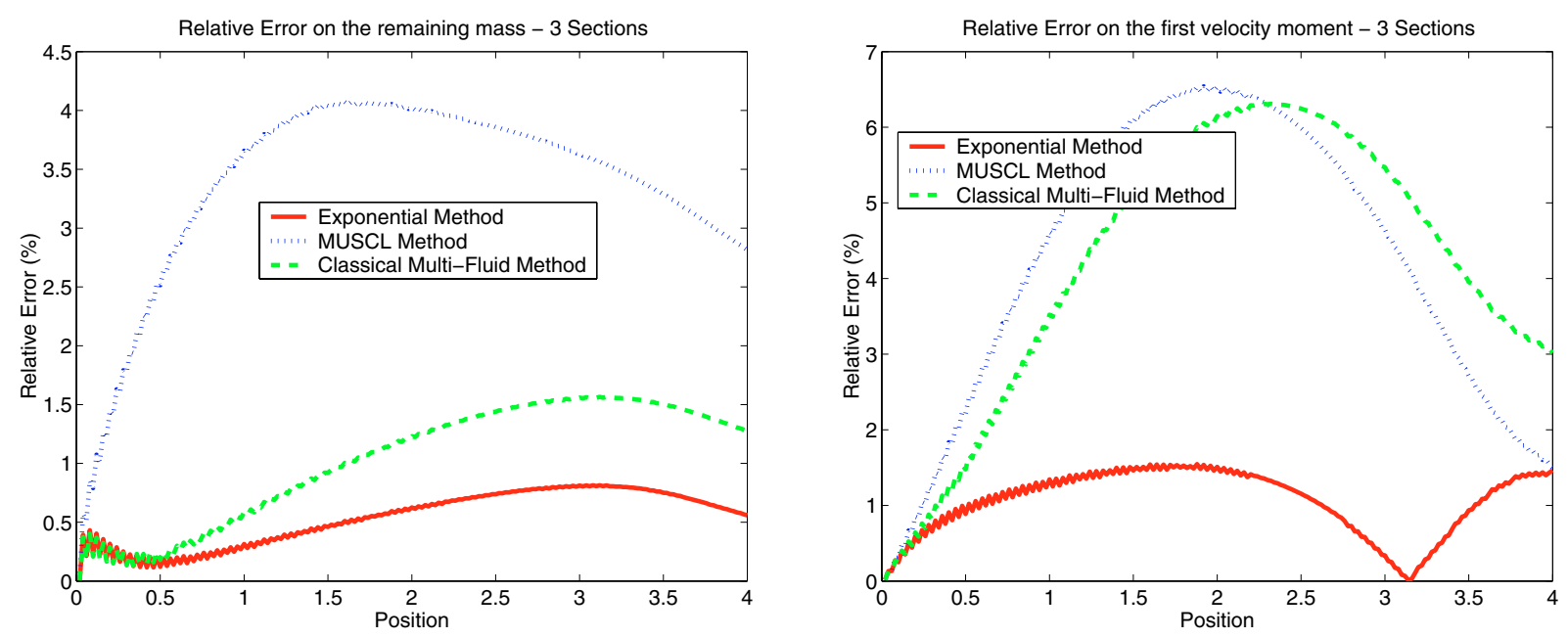

FiguRE 21. Relative errors on mass density and first velocity moment.

for the second-order methods. As expected, the exponential method is the one keeping the best accuracy on every quantity tested. It can also be noted that the classical first-order method gives better results (especially for the mass density) than the second-order MUSCL method.

\section{Conclusion}

In this paper, a way to improve the classical multi-fluid model was presented. This improvement was achieved by taking into account the information on two moments of the number density function in any section. Such an approach resulted in the development of a new model for polydispersed two-phase flows constituted of three conservation equations (number density, mass density and momentum) for any section. To be able to derive the corresponding model, the shape of the number density function has to be prescribed in every section. We proposed here an approximation relying on an exponential distribution and we showed that this choice yields a robust and globally second order accurate numerical scheme that preserves the positivity of the droplet number density and satisfies a maximum principle on the mean droplet mass.

The performed numerical simulations show a real improvement compared to the classical multi-fluid method for high - as well as for very low - numbers of sections. Indeed, for high numbers of sections (for example, 50), the order of accuracy is comparable to the MUSCL method applied to the droplet number density equation. Furthermore, when a low number of sections is used, the importance of preserving two moments is emphasized since the MUSCL method does not provide better results than the classical multi-fluid method whereas the improved one does give the right trend for the evolution of the Sauter mean radius and of the remaining mass density.

The approach presented in this article can be extended to more complex phenomena such as secondary break-up and coalescence whose simulation highlights the necessity to reduce the number of sections in order to remain computationally efficient. Future works and numerical simulations will tend to achieve it.

Acknowledgements. The authors would like to thank Marc Massot for introducing them to the multi-fluid models and for helpful discussions and comments. 


\section{REFERENCES}

[1] M.R. Archambault, C.F. Edwards and R.W. MacCormack, Computation of spray dynamics by moment transport equations I: Theory and development. Atomization Spray 13 (2003) 63-87.

[2] M.R. Archambault, C.F. Edwards and R.W. MacCormack, Computation of spray dynamics by moment transport equations II: Application to calculation of a quasi-one-dimensional spray. Atomization Spray 13 (2003) 89-115.

[3] J.C. Beck and A.P. Watkins, On the development of spray submodels based on droplet size moments. J. Comput. Phys. 182 (2002) 1-36.

[4] J.C. Beck and A.P. Watkins, The droplet number moments approach to spray modeling: The development of heat and mass transfer sub-models. Int. J. Heat Fluid Flow 24 (2003) 242-259.

[5] A.V. Bobylev and T. Ohwada, The error of the splitting scheme for solving evolutionary equations. Appl. Math. Lett. 14 (2001) $45-48$.

[6] F. Bouchut, On zero pressure gas dynamics. Advances in Kinetic Theory and Computing, Selected Papers, Ser. Adv. Math. Appl. Sci. 22 (1994) 171-190.

[7] F. Bouchut, S. Jin and X. Li, Numerical approximations of pressureless and isothermal gas dynamics. SIAM J. Numer. Anal. 41-1 (2003) 135-158.

[8] R. Clift, J.R. Grace and M.E. Weber, Bubbles, drops and particles. Academic Press (1978).

[9] C.T. Crowe, Review - Numerical methods for dilute gas-particle flows. Trans. ASME J. Fluids Eng. 104 (1982) $297-303$.

[10] C. Dafermos, Generalized characteristics in hyperbolic systems of conservation laws. Arch. Rational Mech. Anal. 107 (1989) $127-155$.

[11] K. Domelevo, Analyse mathématique et numérique d'une modélisation cinétique d'un brouillard de gouttelettes dans un écoulement gazeux turbulent. Ph.D. Thesis, École Polytechnique, France (1996).

[12] K. Domelevo, The kinetic sectional approach for noncolliding evaporating sprays. Atomization Spray 11 (2001) $291-303$.

[13] D.A. Drew, Mathematical modeling of two-phase flows. Annu. Rev. Fluid. Mech. 15 (1983) 261-291.

[14] G. Dufour, Un modèle multi-fluide Eulerien pour les écoulements diphasiques à inclusions dispersées. Ph.D. Thesis, Université Toulouse III, France (2005).

[15] J.K. Dukowicz, A particle-fluid numerical model for liquid sprays. J. Comput. Phys. 35 (1980) 229-253.

[16] J. Dupays, Contribution à l'étude du rôle de la phase condensée dans la stabilité d'un propulseur à propergol solide pour lanceur spatial. Ph.D. Thesis, Institut National Polytechnique de Toulouse, France (1996).

[17] H. Grad, On the kinetic theory of rarefied gases. Comm. Pure Appl. Math. 2 (1949) 331-407.

[18] J.B. Greenberg, I. Silverman and Y. Tambour, On the origin of spray sectional conservation equations. Combustion Flame 93 (1993) 90-96.

[19] A. Harten, B. Engquist, S. Osher and S. Chakravarthy, Uniformly high order accurate essentially non-oscillatory schemes III. J. Comput. Phys. 126 (1987) 231-303.

[20] J.J. Hylkema, Modélisation cinétique et simulation numérique d'un brouillard dense de gouttelettes. Applications aux propulseurs à poudre. Ph.D. Thesis, ENSAE, France (1999).

[21] M. Ishii, Thermo-fluid dynamics of two-phase flows. Eyrolles, Paris (1975).

[22] F. Laurent, Analyse numérique d'une méthode multi-fluide Eulérienne pour la description de sprays qui s'évaporent. $C$. $R$. Acad. Sci. Paris Ser. I 334 (2002) 417-422.

[23] F. Laurent, Numerical analysis of Eulerian multi-fluid models in the context of kinetic formulations for dilute evaporating sprays. To be submitted.

[24] F. Laurent and M. Massot, Multi-fluid modeling of laminar poly-disperse spray flames: origin, assumptions and comparison of sectional and sampling methods. Combust. Theor. Model. 5 (2001) 537-572.

[25] F. Laurent, M. Massot and P. Villedieu, Eulerian multi-fluid modeling for the numerical simulation of coalescence in polydisperse dense liquid sprays. J. Comput. Phys. 194 (2004) 505-543.

[26] F. Laurent, V. Santoro, M. Noskov, M.D. Smooke, A. Gomez and M. Massot, Accurate treatment of size distribution effects in polydisperse spray diffusion flames: multi-fluid modelling, computations and experiments. Combust. Theor. Model. 8 (2004) $385-412$.

[27] C.D. Levermore, Moment closure hierarchies for kinetics theories. J. Statist. Phys. 83 (1996) 1021-1065.

[28] D. Levy, G. Puppo and G. Russo, On the behavior of the total variation in CWENO methods for conservation laws. Appl. Numer. Math. 33 (2000) 407-414.

[29] R. Maxey and J. Riley, Equation of motion of a small rigid sphere in a non-unifom flow. Phys. Fluids 26 (1983) 883-889.

[30] P.J. O'Rourke, Collective drop effects on vaporizing liquid sprays. Ph.D. Thesis, Los Alamos national Laboratory, New Mexico 87545 (1981).

[31] F. Poupaud and M. Rascle, Measure solutions to the linear multi-dimensional transport equation with non-smooth coefficients. Comm. P.D.E 22 (1997) 337-358.

[32] D. Ramkrishna and A.G. Fredrickson, Population balances: Theory and applications to particulate systems in engineering. Academic Press (2000). 
[33] M. Rüger, S. Hohmann, M. Sommerfeld and G. Kohnen, Euler-Lagrange calculations of turbulent sprays: the effect of droplet collisions and coalescence. Atomization Spray 10 (2000).

[34] O. Simonin, Modélisation numérique des écoulements turbulents diphasiques à inclusions dispersées. École de Printemps de Mécanique des Fluides numériques, Aussois (1991).

[35] O. Simonin, Continuum modeling of dispersed two-phase flows. Combustion and turbulence in two-phase flows. Lecture Series 1996-02, Von Karman Inst. for fluid dyn. (1996).

[36] G. Strang, On the construction and comparison of difference schemes. SIAM J. Numer. Anal. 5 (1968) 507-517.

[37] Y. Tambour, A Lagrangian sectional approach for simulating droplet size distribution of vaporizing fuel sprays in a turbulent jet. Combustion Flame 60 (1985) 15-28.

[38] B. Van Leer, A second-order sequel to Godunov's method. J. Comput. Phys. 32 (1979) $101-136$.

[39] F.A. Williams, Spray combustion and atomization. Phys. Fluids 1 (1958) 541-555.

[40] F.A. Williams, Combustion Theory. Addison-Wesley Publishing (1985).

[41] D.L. Wright, R. McGraw and D.E. Rosner, Bivariate extension of the quadrature method of moments for modeling simultaneous coagulation and sintering of particle populations. J. Colloid Interf. Sci. 236 (2001) 242-251.

To access this journal online: www.edpsciences.org 